\title{
The Regularization of the Divergent Integrals in 2-D Elastostatics
}

\author{
V.V. Zozulya \\ Centro de Investigacion Cientifica de Yucatan A.C., \\ Calle 43, No 130, Colonia: Chuburná de Hidalgo, C.P. 97200, \\ Mérida, Yucatán, México. E-mail: zozulya@cicy.mx
}

\begin{abstract}
This article considers weakly singular, singular and hypersingular integrals, which arise when the boundary integral equation (BIE) methods are used to solve problems in 2-D elastostatics. For their regularization, an approach based on the theory of distribution and application of the Green's theorem has been used. The expressions, which allow an easy calculation of the weakly singular, singular and hypersingular integrals for straight and curved boundary elements, have been constructed.
\end{abstract}

Key words: weakly singular, singular, hypersingular integrals, regularization, boundary integral equations

\section{Introduction}

The BIE is a very powerful tool for the solution of mathematical problems in science and engineering [1-3]. When the BIE are solved numerically divergent integrals have to be calculated. Numerical methods developed for the regular integrals calculation cannot be used for their calculation. There are many methods for the divergent integral calculation (for references, see books $[3,7]$ and review articles $[4,5,8]$ and references there). We will not discuss here advantages and disadvantages of these methods; they have already been done in the above mentioned reviews. We will consider here method of the divergent integral regularization developed in [10-16] in more details. This method is based on the Green's theorem application in the sense of the theory of distributions. For the first time this approach has been used in [10]. Then, it was further developed in [15] and [16] for the regularization of the hypersingular integrals in static and dynamic problems of fracture mechanics. More applications of this regularization method can be found in review articles [5,6]. The equations presented in [11, 12, 14] permit to transform divergent integrals to the regular ones. They can be applied for a wide class of divergent integral regularizations.

In the present paper, the above mentioned approach for the divergent integral regularization is developed and applied for the case of 2-D elastostatic problems. The weakly singular (WS) integrals are treated as improper, the singular integrals are treated in the sense of the Cauchy principal value $(P V)$, and the hypersingular integrals are treated as the Hadamard's finite part $(F P)$ integrals, and the same method for their regularization is used. Straight and curved boundary elements $(\mathrm{BE})$ with different number 
of nodal points are considered. The regularized equations for the calculation of the $W S$, $P V$ and FP 1-D integrals have been presented here.

\section{Problem Statement and the BIE}

Let us consider a homogeneous, lineally elastic body, which in 2-D Euclidean space $\mathfrak{R}^{2}$ occupies an open bounded region $V$ with $C^{0,1}$ Lipschitzian regular boundary $\partial V$. The boundary contains two parts $\partial V_{u}$ and $\partial V_{p}$ such that $\partial V_{u} \cap \partial V_{p}=\varnothing$ and $\partial V_{u} \cup \partial V_{p}=\partial V$. On part $\partial V_{u}$ the displacement $u_{i}(\mathbf{x})$ and on part $\partial V_{p}$ the traction $p_{i}(\mathbf{x})$ are prescribed, respectively. The body may by affected by the volume force $b_{i}(\mathbf{x})$. We assume that the displacement and their gradients are small. In this case the differential equations of the equilibrium in the displacement may be presented in the form

$$
A_{i j} u_{j}+b_{i}=0, \quad A_{i j}=\mu \delta_{i j} \partial_{k} \partial_{k}+(\lambda+\mu) \partial_{i} \partial_{j} . \quad \forall \mathbf{x} \in V
$$

If the body occupies a finite region $V$, it is necessary to establish the boundary conditions. The mixed boundary conditions are

$$
\begin{gathered}
u_{i}(\mathbf{x})=\varphi_{i}(\mathbf{x}), \quad \forall \mathbf{x} \in \partial V_{u}, \\
p_{i}(\mathbf{x})=\sigma_{i j}(\mathbf{x}) n_{j}(\mathbf{x})=P_{i j}\left[u_{j}(\mathbf{x})\right]=\psi_{i}(\mathbf{x}) . \quad \forall \mathbf{x} \in \partial V_{p}
\end{gathered}
$$

The differential operator $P_{i j}: u_{j} \rightarrow p_{i}$ is called the stress operator. It transforms the displacements into the tractions. For the homogeneous isotropic elastic medium it has the form

$$
P_{i j}=\lambda n_{i} \partial_{k}+\mu\left(\delta_{i j} \partial_{n}+n_{k} \partial_{i}\right)
$$

Here $n_{i}$ are the components of the outward unit normal vector, $\partial_{n}=n_{i} \partial_{i}$ is the derivative in the direction of the vector $\mathbf{n}(\mathbf{x})$ normal to the surface $\partial V$.

If the equation (2.1) is defined in an infinite region, then its solution must satisfy additional conditions at infinity

$$
u_{i}(\mathbf{x})=O\left(\ln \left(r^{-1}\right)\right), \sigma_{i j}(\mathbf{x})=O\left(r^{-1}\right) \text { for } r \rightarrow \infty .
$$

The boundary value problem of the elastostatics (2.1)-(2.2), can be transformed to the boundary integral equations. In order to do that the boundary integral representations for the displacement and the traction have usually been used. On the smooth part of the boundary these representations have the form

$$
\begin{aligned}
& \pm \frac{1}{2} u_{i}(\mathbf{x})=U_{i}(\mathbf{p}, \mathbf{x}, \partial V)-W_{i}(\mathbf{u}, \mathbf{x}, \partial V)+U_{i}(\mathbf{f}, \mathbf{x}, V) \\
& \pm \frac{1}{2} p_{i}(\mathbf{x})=K_{i}(\mathbf{p}, \mathbf{x}, \partial V)-F_{i}(\mathbf{u}, \mathbf{x}, \partial V)+K_{i}(\mathbf{f}, \mathbf{x}, V) .
\end{aligned}
$$

Here the plus and minus signs are used for the interior and exterior problems, respectively. For convenience and compactness of the BIE consideration, we introduce the following elastostatic potentials 


$$
\begin{array}{r}
U_{i}(\mathbf{p}, \mathbf{y}, \partial V)=\int_{\partial V} p_{j}(\mathbf{x}) U_{i j}(\mathbf{x}-\mathbf{y}) d S, W_{i}(\mathbf{u}, \mathbf{y}, \partial V)=\int_{\partial V} u_{j}(\mathbf{x}, k) W_{i j}(\mathbf{x}, \mathbf{y}) d S, \\
K_{i}(\mathbf{p}, \mathbf{y}, \partial V)=\int_{\partial V} p_{j}(\mathbf{x}) K_{i j}(\mathbf{x}, \mathbf{y}) d S, F_{i}(\mathbf{u}, \mathbf{y}, \partial V)=\int_{\partial V} u_{j}(\mathbf{x}, k) F_{i j}(\mathbf{x}, \mathbf{y}) d S, \\
U_{i}(\mathbf{f}, \mathbf{y}, V)=\int_{V} f_{j}(\mathbf{x}) U_{i j}(\mathbf{x}-\mathbf{y}) d V, K_{i}(\mathbf{f}, \mathbf{y}, V)=\int_{V} f_{j}(\mathbf{x}) K_{i j}(\mathbf{x}, \mathbf{y}) d V .
\end{array}
$$

The kernels here are the fundamental solutions for the differential operator $A_{i j}$. They have the form

$$
\begin{gathered}
U_{i j}(\mathbf{x}-\mathbf{y})=\frac{1}{8 \pi \mu(1-v)}\left((3-4 v) \delta_{i j} \ln \frac{1}{r}+\partial_{i} r \partial_{j} r\right), \\
W_{i j}(\mathbf{x}, \mathbf{y})=\frac{-1}{4 \pi(1-v) r}\left(n_{k}(\mathbf{x}) \partial_{k} r\left((1-2 v) \delta_{i j}+2 \partial_{i} r \partial_{j} r\right)+(1-2 v)\left(n_{i}(\mathbf{x}) \partial_{j} r-n_{j}(\mathbf{x}) \partial_{i} r\right)\right), \\
K_{i j}(\mathbf{x}, \mathbf{y})=\frac{1}{4 \pi(1-v) r}\left(n_{k}(\mathbf{y}) \partial_{k} r\left((1-2 v) \delta_{i j}+2 \partial_{i} r \partial_{j} r\right)+(1-2 v)\left(n_{i}(\mathbf{y}) \partial_{j} r-n_{j}(\mathbf{y}) \partial_{i} r\right)\right), \\
F_{i j}(\mathbf{x}, \mathbf{y})=\frac{\mu}{2 \pi(1-v) r^{2}}\left(2 n _ { k } ( \mathbf { x } ) \partial _ { k } r \left((1-2 v) n_{i}(\mathbf{y}) \partial_{j} r+v\left(\delta_{i j} n_{k}(\mathbf{y}) \partial_{k} r+n_{j}(\mathbf{y}) \partial_{i} r\right)-\right.\right. \\
\left.-4 n_{k}(\mathbf{y}) \partial_{k} r \partial_{i} r \partial_{j} r\right)+2 v\left(n_{i}(\mathbf{x}) n_{k}(\mathbf{y}) \partial_{k} r \partial_{j} r+n_{k}(\mathbf{x}) n_{k}(\mathbf{y}) \partial_{i} r \partial_{j} r\right)+ \\
\left.+(1-2 v)\left(2 n_{j}(\mathbf{x}) n_{k}(\mathbf{y}) \partial_{k} r \partial_{i} r+n_{k}(\mathbf{x}) n_{k}(\mathbf{y}) \delta_{i k}+n_{i}(\mathbf{x}) n_{j}(\mathbf{y})\right)-(1-4 v) n_{j}(\mathbf{x}) n_{i}(\mathbf{y})\right) .
\end{gathered}
$$

Here $r=\sqrt{\left(x_{1}-y_{1}\right)^{2}+\left(x_{2}-y_{2}\right)^{2}}$ is the distance between points $\mathbf{x}$ and $\mathbf{y}$ in 2-D Euclidean space $\mathfrak{R}^{2}, \partial_{i} r=\frac{\partial r}{\partial x_{i}}=-\frac{\partial r}{\partial y_{i}}=\frac{x_{i}-y_{i}}{r}$ are derivatives with respect to $x_{i}$ and $y_{i}$.

Together with boundary conditions, the integral representations (2.5) are used for the creation of the BIE that correspond to the boundary value problems of elastostatics. Using (2.5) we can construct various BIEs for the same boundary value problem. For example, if we use the first and the second boundary integral representations on the parts $\partial V_{u}$ and $\partial V_{p}$ of the boundary, the BIEs have the form

$$
\begin{aligned}
& \frac{1}{2} u_{i}(\mathbf{x})-U_{i}\left(\mathbf{p}, \mathbf{x}, \partial V_{u}\right)+W_{i}\left(\mathbf{u}, \mathbf{x}, \partial V_{p}\right)=\Phi_{i}(\mathbf{x}), \quad \forall \mathbf{x} \in \partial V_{u} \\
& \left.\frac{1}{2} p_{i}(\mathbf{x})-K_{i}\left(\mathbf{p}, \mathbf{x}, \partial V_{u}\right)+F_{i}\left(\mathbf{u}, \mathbf{x}, \partial V_{p}\right)\right)=\Psi_{i}(\mathbf{x}), \quad \forall \mathbf{x} \in \partial V_{p}
\end{aligned}
$$

where

$$
\begin{aligned}
& \Phi_{i}(\mathbf{x})=U_{i}(\mathbf{f}, \mathbf{x}, V)+U_{i}\left(\psi, \mathbf{x}, \partial V_{p}\right)-W_{i}\left(\varphi, \mathbf{x}, \partial V_{p}\right), \\
& \Psi_{i}(\mathbf{x})=K_{i}(\mathbf{f}, \mathbf{x}, V)+K_{i}\left(\psi, \mathbf{x}, \partial V_{p}\right)-F_{i}\left(\varphi, \mathbf{x}, \partial V_{p}\right) .
\end{aligned}
$$

These BIEs are not unique. Many other BIEs that correspond to the boundary value problems of elastostatics have been considered in [1-3]. 
The kernels (2.6) contain singularities of different kind; the kernel $U_{i j}(\mathbf{x}-\mathbf{y})$ is weakly singular, $W_{i j}(\mathbf{x}, \mathbf{y})$ and $K_{i j}(\mathbf{x}, \mathbf{y})$ are singular and $F_{i j}(\mathbf{x}, \mathbf{y})$ is hypersingular. The integrals with singularities can not be considered in the usual (Riemann or Lebegue) sense. In order for such integrals to have sense, it is necessary to carry out a special consideration.

Definition 2.1. Integrals in (2.7) with kernels $U_{i j}(\mathbf{x}-\mathbf{y})$ are weakly singular and must be considered as improper

$$
W . S . \int_{\partial V} p_{i}(\mathbf{x}) U_{i j}(\mathbf{x}-\mathbf{y}) d S=\lim _{\varepsilon \rightarrow 0} \int_{\partial V \backslash \partial V_{\varepsilon}} p_{i}(\mathbf{x}) U_{i j}(\mathbf{x}-\mathbf{y}) d S .
$$

Here $\partial V_{\varepsilon}$ is a part of the boundary, projection of which on the tangential plane is contained in the circle $C_{\varepsilon}(\mathbf{x})$ of radius $\varepsilon$ with center at $\mathbf{x}$.

Definition 2.2. Integrals in (2.7) with kernels $W_{i j}(\mathbf{x}, \mathbf{y})$ and $K_{i j}(\mathbf{x}, \mathbf{y})$ are singular and must be considered in the sense of the Cauchy principal values as

$$
\begin{aligned}
& P . V \cdot \int_{\partial V} u_{i}(\mathbf{x}) W_{i j}(\mathbf{x}, \mathbf{y}) d S=\lim _{\varepsilon \rightarrow 0} \int_{\partial V \backslash \partial V(r<\varepsilon)} u_{i}(\mathbf{x}) W_{i j}(\mathbf{x}, \mathbf{y}) d S, \\
& P . V . \int_{\partial V} p_{i}(\mathbf{x}) K_{i j}(\mathbf{x}, \mathbf{y}) d S=\lim _{\varepsilon \rightarrow 0} \int_{\partial V \backslash \partial V(r<\varepsilon)} p_{i}(\mathbf{x}) K_{i j}(\mathbf{x}, \mathbf{y}) d S .
\end{aligned}
$$

Here $\partial V(r<\varepsilon)$ is a part of the boundary, projection of which on the tangential plane is the circle $C_{\varepsilon}(\mathbf{x})$ of radius $\varepsilon$ with center at $\mathbf{x}$.

Definition 2.3. Integrals in (2.7) with kernels $F_{i j}(\mathbf{x}, \mathbf{y})$ are hypersingular and must be considered in the sense of the Hadamard finite part as

$$
F . P . \int_{\partial V} u_{i}(\mathbf{x}) F_{j i}(\mathbf{x}-\mathbf{y}) d S=\lim _{\varepsilon \rightarrow 0}\left(\int_{\partial V \backslash \partial V(r<\varepsilon)} u_{i}(\mathbf{x}) W_{j i}(\mathbf{x}-\mathbf{y}) d S+2 u_{j}(\mathbf{x}) \frac{f_{j}(\mathbf{x})}{\partial V(r<\varepsilon)}\right) .
$$

Here functions $f_{j}(\mathbf{x})$ are chosen to assure the existence of the limit.

We will apply these definitions to the integrals in (2.7) and will investigate their singularities and develop methods for the calculation of these divergent integrals.

The BIE is usually solved numerically, transforming them into a discrete system of finite dimensional equations. This method of the BIE solution is called the boundary element method (BEM). 


\section{BEM Equations}

To transform the BIE into the finite dimensional BEM equations we have to split the boundary $\partial V$ into a collection of finite boundary elements (BE)

$$
\partial V=\bigcup_{n=1}^{N} \partial V_{n}, \quad \partial V_{n} \cap \partial V_{k}=\varnothing, \quad \text { if } n \neq k .
$$

On each BE we shall choose $Q$ nodes of interpolation and the shape functions $\varphi_{n q}(\mathbf{x})$. Then the displacement and traction on each BE $\partial V_{n}$ will be approximately represented in the form

$$
\begin{aligned}
& u_{i}(\mathbf{x}) \approx \sum_{q=1}^{Q} u_{i}^{n}\left(\mathbf{x}_{q}\right) \varphi_{n q}(\mathbf{x}), \quad \mathbf{x} \in \partial V_{n}, \\
& p_{i}(\mathbf{x}) \approx \sum_{q=1}^{Q} p_{i}^{n}\left(\mathbf{x}_{q}\right) \varphi_{n q}(\mathbf{x}) \mathbf{x} \in \partial V_{n},
\end{aligned}
$$

and on the whole boundary $\partial V$ in the form

$$
\begin{aligned}
& u_{i}(\mathbf{x}) \approx \sum_{n=1}^{N} \sum_{q=1}^{Q} u_{i}^{n}\left(\mathbf{x}_{q}\right) \varphi_{n q}(\mathbf{x}), \quad \mathbf{x} \in \bigcup_{n=1}^{N} \partial V_{n}, \\
& p_{i}(\mathbf{x}) \approx \sum_{n=1}^{N} \sum_{q=1}^{Q} p_{i}^{n}\left(\mathbf{x}_{q}\right) \varphi_{n q}(\mathbf{x}), \quad \mathbf{x} \in \bigcup_{n=1}^{N} \partial V_{n} .
\end{aligned}
$$

Here and below if the node $q$ belongs to several BEs it is considered only once in these sums.

Substitution of the expressions (3.3) in (2.4) gives us the finite-dimensional representations for the displacements and traction vectors on the boundary in the form

$$
\begin{aligned}
& \frac{1}{2} u_{i}^{m}\left(\mathbf{y}_{r}\right)=\sum_{n=1}^{N} \sum_{q=1}^{Q}\left[U_{j i}^{n}\left(\mathbf{y}_{r}, \mathbf{x}_{q}\right) p_{j}^{n}\left(\mathbf{x}_{q}\right)-W_{j i}^{n}\left(\mathbf{x}_{r}, \mathbf{x}_{q}\right) u_{j}^{n}\left(\mathbf{x}_{q}\right)+U_{i}\left(\mathbf{f}, \mathbf{y}, V_{n}\right),\right. \\
& \frac{1}{2} p_{i}^{m}\left(\mathbf{y}_{r}\right)=\sum_{n=1}^{N} \sum_{q=1}^{Q}\left[K_{j i}^{n}\left(\mathbf{y}_{r}, \mathbf{x}_{q}\right) p_{j}^{n}\left(\mathbf{y}_{q}\right)-F_{j i}^{n}\left(\mathbf{y}_{r}, \mathbf{x}_{q}\right) u_{j}^{n}\left(\mathbf{x}_{q}\right)+K_{i}\left(\mathbf{f}, \mathbf{y}, V_{n}\right),\right.
\end{aligned}
$$

where

$$
\begin{aligned}
& U_{j i}^{n}\left(\mathbf{y}_{r}, \mathbf{x}_{q}\right)=\int_{\partial V_{n}} U_{j i}\left(\mathbf{y}_{r}, \mathbf{x}\right) \varphi_{n q}(\mathbf{x}) d S, \quad W_{j i}^{n}\left(\mathbf{y}_{r}, \mathbf{x}_{q}\right)=\int_{\partial V_{n}} W_{j i}\left(\mathbf{y}_{r}, \mathbf{x}\right) \varphi_{n q}(\mathbf{x}) d S, \\
& K_{j i}^{n}\left(\mathbf{y}_{r}, \mathbf{x}_{q}\right)=\int_{\partial V_{n}} K_{j i}\left(\mathbf{y}_{r}, \mathbf{x}\right) \varphi_{n q}(\mathbf{x}) d S, \quad F_{j i}^{n}\left(\mathbf{y}_{r}, \mathbf{x}_{q}\right)=\int_{\partial V_{n}} F_{j i}\left(\mathbf{y}_{r}, \mathbf{x}\right) \varphi_{n q}(\mathbf{x}) d S .
\end{aligned}
$$

The volume potentials $U_{i}\left(\mathbf{f}, \mathbf{y}, V_{n}\right)$ and $K_{i}\left(\mathbf{f}, \mathbf{y}, V_{n}\right)$ depend on the discretization of the domain $V$. More detailed information about discretization, interpolation and transition from the BIE to the BEM equations can be found in [1-3]. 


\section{Boundary Elements and Approximation in the General Case}

In order to calculate integrals in (3.5) let us introduce the local coordinate $\xi$ on the BEs. Then global coordinates can be expressed in the form

$$
x_{i}(\xi)=\sum_{q=0}^{Q} x_{i}^{q} \varphi_{n q}(\xi), y_{i}(\xi)=\sum_{q=0}^{Q} y_{i}^{q} \varphi_{n q}(\xi) .
$$

Here $x_{i}^{q}$ and $y_{i}^{q}$ are the global coordinates of the nodal points and $\varphi_{n q}(\xi)$ shape or interpolation functions. Since we will use the same shape functions on each BE, index $n$ will be omitted in $\varphi_{n q}(\xi)$. For the BE with $Q+1$ nodes of interpolation an approximation polynomial (shape function) of degree $Q$ is used. They are given by

$$
\varphi_{q}(\xi)=\frac{\left(\xi-\xi_{0}\right)\left(\xi-\xi_{1}\right) \ldots\left(\xi-\xi_{q-1}\right)\left(\xi-\xi_{q+1}\right) \ldots\left(\xi-\xi_{Q}\right)}{\left(\xi_{q}-\xi_{0}\right)\left(\xi_{q}-\xi_{1}\right) \ldots\left(\xi_{q}-\xi_{q-1}\right)\left(\xi_{q}-\xi_{q+1}\right) \ldots\left(\xi_{q}-\xi_{Q}\right)} .
$$

The distance between points $\mathbf{x}$ and $\mathbf{y}$ in local coordinates is

$$
r(\xi, \zeta)=\sqrt{\left(\left(x_{1}^{q} \varphi_{q}(\xi)-y_{1}^{r} \varphi_{r}(\zeta)\right)^{2}+\left(x_{2}^{q} \varphi_{q}(\xi)-y_{2}^{r} \varphi_{r}(\zeta)\right)^{2}\right.},
$$

where $x_{i}(\xi)=\sum_{q=0}^{Q} x_{i}^{q} \varphi_{q}(\xi)=x_{i}^{q} \varphi_{q}(\xi) y_{i}(\zeta)=\sum_{q=0}^{Q} y_{i}^{q} \varphi_{q}(\zeta)=y_{i}^{q} \varphi_{q}(\zeta)$.

Curved 1-D element $d S$ in (3.5) in the local coordinates has the form

$$
d S=J(\xi) d \xi, J(\xi)=\sqrt{\left(d x_{1}(\xi) / d \xi\right)^{2}+\left(d x_{2}(\xi) / d \xi\right)^{2}} .
$$

Jacobian here may be also presented in the form

$$
J(\xi)=\left[\left(\sum_{q=0}^{Q} x_{1}^{q} \frac{d \varphi_{q}(\xi)}{d \xi}\right)^{2}+\left(\sum_{q=0}^{Q} x_{2}^{q} \frac{d \varphi_{q}(\xi)}{d \xi}\right)^{2}\right]^{1 / 2} .
$$

Analysis of the fundamental solutions in (2.6) shows that integrals in (3.5) contain kernels with different singularities as $\mathbf{x} \rightarrow \mathbf{y}$. In order to construct the BEM equations we have to calculate the divergent integrals related to the following fundamental solutions:

1. Weakly singular (WS) :

$$
\begin{aligned}
& U_{11}(\mathbf{x}, \mathbf{y})=\frac{1}{8 \pi \mu(1-v)}\left((3-4 v) \ln \frac{1}{r}+\frac{x_{1}^{2}}{r^{2}}\right), \\
& U_{22}(\mathbf{x}, \mathbf{y})=\frac{1}{8 \pi \mu(1-v)}\left((3-4 v) \ln \frac{1}{r}+\frac{x_{2}{ }^{2}}{r^{2}}\right),
\end{aligned}
$$




$$
U_{12}(\mathbf{x}, \mathbf{y})=U_{21}(\mathbf{x}, \mathbf{y}) \frac{1}{8 \pi \mu(1-v)} \frac{x_{1} x_{2}}{r^{2}} .
$$

2. Singular in the sense of the principle value $(P V)$ :

$$
\begin{aligned}
& W_{11}(\mathbf{x}, \mathbf{y})=-\frac{(1-2 v)}{4 \pi(1-v)}\left(n_{1}(\mathbf{x}) \frac{x_{1}}{r^{2}}+n_{2}(\mathbf{x}) \frac{x_{2}}{r^{2}}\right)-\frac{1}{2 \pi(1-v)}\left(n_{1}(\mathbf{x}) \frac{x_{1}^{3}}{r^{4}}+n_{2}(\mathbf{x}) \frac{x_{1}{ }^{2} x_{2}}{r^{4}}\right), \\
& W_{12}(\mathbf{x}, \mathbf{y})=\frac{-1}{2 \pi(1-v)}\left(n_{1}(\mathbf{x}) \frac{x_{1}^{2} x_{2}}{r^{4}}+n_{2}(\mathbf{x}) \frac{x_{2}^{2} x_{1}}{r^{4}}\right)+\frac{(1-2 v)}{4 \pi(1-v)}\left(n_{1}(\mathbf{x}) \frac{x_{2}}{r^{2}}-n_{2}(\mathbf{x}) \frac{x_{1}}{r^{2}}\right), \\
& W_{21}(\mathbf{x}, \mathbf{y})=\frac{-1}{2 \pi(1-v) r}\left(n_{1}(\mathbf{x}) \frac{x_{1}^{2} x_{2}}{r^{4}}+n_{2}(\mathbf{x}) \frac{x_{2}^{2} x_{1}}{r^{4}}\right)+\frac{(1-2 v)}{4 \pi(1-v)}\left(n_{2}(\mathbf{x}) \frac{x_{1}}{r^{2}}-n_{1}(\mathbf{x}) \frac{x_{2}}{r^{2}}\right), \\
& W_{22}(\mathbf{x}, \mathbf{y})=-\frac{(1-2 v)}{4 \pi(1-v)}\left(n_{1}(\mathbf{x}) \frac{x_{1}}{r^{2}}+n_{2}(\mathbf{x}) \frac{x_{2}}{r^{2}}\right)-\frac{1}{2 \pi(1-v)}\left(n_{2}(\mathbf{x}) \frac{x_{2}{ }^{3}}{r^{4}}+n_{1}(\mathbf{x}) \frac{x_{2}{ }^{2} x_{1}}{r^{4}}\right), \\
& K_{11}(\mathbf{x}, \mathbf{y})=\frac{(1-2 v)}{4 \pi(1-v)}\left(n_{1}(\mathbf{y}) \frac{x_{1}}{r^{2}}+n_{2}(\mathbf{y}) \frac{x_{2}}{r^{2}}\right)+\frac{1}{2 \pi(1-v)}\left(n_{1}(\mathbf{y}) \frac{x_{1}^{3}}{r^{4}}+n_{2}(\mathbf{y}) \frac{x_{1}^{2} x_{2}}{r^{4}}\right), \\
& K_{12}(\mathbf{x}, \mathbf{y})=\frac{1}{2 \pi(1-v) r}\left(n_{1}(\mathbf{y}) \frac{x_{1}}{r}+n_{2}(\mathbf{y}) \frac{x_{2}}{r}\right) \frac{x_{1} x_{2}}{r^{2}}+\frac{(1-2 v)}{4 \pi(1-v) r}\left(n_{1}(\mathbf{y}) \frac{x_{2}}{r}-n_{2}(\mathbf{y}) \frac{x_{1}}{r}\right), \\
& K_{21}(\mathbf{x}, \mathbf{y})=\frac{1}{2 \pi(1-v) r}\left(n_{1}(\mathbf{y}) \frac{x_{1}}{r}+n_{2}(\mathbf{y}) \frac{x_{2}}{r}\right) \frac{x_{1} x_{2}}{r^{2}}+\frac{(1-2 v)}{4 \pi(1-v) r}\left(n_{2}(\mathbf{y}) \frac{x_{1}}{r}-n_{1}(\mathbf{y}) \frac{x_{2}}{r}\right), \\
& K_{22}(\mathbf{x}, \mathbf{y})=\frac{(1-2 v)}{4 \pi(1-v) r}\left(n_{1}(\mathbf{y}) \frac{x_{1}}{r}+n_{2}(\mathbf{y}) \frac{x_{2}}{r}\right)+\frac{1}{2 \pi(1-v)}\left(n_{2}(\mathbf{y}) \frac{x_{2}^{3}}{r^{4}}+n_{1}(\mathbf{y}) \frac{x_{1} x_{2}^{2}}{r^{4}}\right) .
\end{aligned}
$$

3. Hypersingular in the sense of the finite part $(F P)$ :

$$
\begin{aligned}
& F_{11}(\mathbf{x}, \mathbf{y})=\frac{\mu}{\pi(1-v)}\left(n_{1}(\mathbf{x}) n_{1}(\mathbf{y}) \frac{x_{1}^{2}}{r^{4}}+\left(n_{1}(\mathbf{y}) n_{2}(\mathbf{x})+n_{2}(\mathbf{y}) n_{1}(\mathbf{x})\right) \frac{x_{1} x_{2}}{2 r^{4}}+\left(n_{1}(\mathbf{y}) n_{1}(\mathbf{x})+n_{2}(\mathbf{y}) n_{2}(\mathbf{x})\right) \frac{1}{2 r^{2}}-\right. \\
& \left.-4 n_{1}(\mathbf{x}) n_{1}(\mathbf{y}) \frac{x_{1}^{4}}{r^{6}}-4 n_{1}(\mathbf{x}) n_{2}(\mathbf{y}) \frac{x_{1}^{3} x_{2}}{r^{6}}-4 n_{2}(\mathbf{x}) n_{1}(\mathbf{y}) \frac{x_{1}^{3} x_{2}}{r^{6}}-4 n_{2}(\mathbf{x}) n_{1}(\mathbf{y}) \frac{x_{1}^{2} x_{2}^{2}}{r^{6}}\right), \\
& F_{12}(\mathbf{x}, \mathbf{y})=F_{21}(\mathbf{x}, \mathbf{y})=\frac{\mu}{\pi(1-v)}\left(\left(n_{1}(\mathbf{y}) n_{1}(\mathbf{x})+n_{2}(\mathbf{y}) n_{2}(\mathbf{x})\right) \frac{x_{1} x_{2}}{2 r^{4}}+\left(n_{1}(\mathbf{y}) n_{2}(\mathbf{x})+n_{2}(\mathbf{y}) n_{1}(\mathbf{x})\right) \frac{1}{2 r^{2}}-\right. \\
& \left.-4 n_{1}(\mathbf{x}) n_{1}(\mathbf{y}) \frac{x_{1}^{3} x_{2}}{r^{6}}-4 n_{1}(\mathbf{x}) n_{2}(\mathbf{y}) \frac{x_{1}^{2} x_{2}^{2}}{r^{6}}-4 n_{2}(\mathbf{x}) n_{1}(\mathbf{y}) \frac{x_{1}^{2} x_{2}^{2}}{r^{6}}-4 n_{2}(\mathbf{x}) n_{1}(\mathbf{y}) \frac{x_{1} x_{2}^{3}}{r^{6}}\right), \\
& F_{22}(\mathbf{x}, \mathbf{y})=\frac{\mu}{\pi(1-v)}\left(n_{2}(\mathbf{x}) n_{2}(\mathbf{y}) \frac{x_{2}^{2}}{r^{4}}+\left(n_{1}(\mathbf{y}) n_{2}(\mathbf{x})+n_{2}(\mathbf{y}) n_{1}(\mathbf{x})\right) \frac{x_{1} x_{2}}{2 r^{4}}+\left(n_{1}(\mathbf{y}) n_{1}(\mathbf{x})+n_{2}(\mathbf{y}) n_{2}(\mathbf{x})\right) \frac{1}{2 r^{2}}-\right. \\
& \left.-4 n_{1}(\mathbf{x}) n_{1}(\mathbf{y}) \frac{x_{1}^{2} x_{2}^{2}}{r^{6}}-4 n_{1}(\mathbf{x}) n_{2}(\mathbf{y}) \frac{x_{1} x_{2}^{3}}{r^{6}}-4 n_{2}(\mathbf{x}) n_{1}(\mathbf{y}) \frac{x_{1} x_{2}^{3}}{r^{6}}-4 n_{2}(\mathbf{x}) n_{1}(\mathbf{y}) \frac{x_{2}^{4}}{r^{6}}\right) .
\end{aligned}
$$


For simplicity we set $\mathbf{y}=0$ in the above equations. Definition of the weakly singular, singular in the sense of the principle value and hypersingular in the sense of the finite part integrals may be found in [5].

We need also to calculate components of the normal vector $n_{1}(\mathbf{x})$ and $n_{2}(\mathbf{x})$ in the local coordinates. For that purpose we will use representations

$$
\mathbf{n}=n_{1} \mathbf{e}_{1}+n_{2} \mathbf{e}_{2}, \boldsymbol{\tau}=\frac{d x_{1}}{d \xi} \mathbf{e}_{1}+\frac{d x_{2}}{d \xi} \mathbf{e}_{2} .
$$

where

$$
n_{1}=\frac{-1}{J(\xi)} \frac{d x_{2}}{d \xi}, n_{2}=\frac{1}{J(\xi)} \frac{d x_{1}}{d \xi}
$$

Using the relation

$$
\frac{d x_{i}(\xi)}{d \xi}=\frac{d}{d \xi} \sum_{q=0}^{Q} x_{i}^{q} \varphi_{q}(\xi)=\sum_{q=0}^{Q} x_{i}^{q} \frac{d \varphi_{q}(\xi)}{d \xi}
$$

the normal and tangential vectors can be written as

$$
\begin{gathered}
\mathbf{n}(\xi)=\frac{-1}{J(\xi)} \sum_{q=0}^{Q} x_{2}^{q} \frac{d \varphi_{q}(\xi)}{d \xi} \mathbf{e}_{1}+\frac{1}{J(\xi)} \sum_{q=0}^{Q} x_{2}^{q} \frac{d \varphi_{q}(\xi)}{d \xi} \mathbf{e}_{2}, \\
\boldsymbol{\tau}(\xi)=\sum_{q=0}^{Q} x_{1}^{q} \frac{d \varphi_{q}(\xi)}{d \xi} \mathbf{e}_{1}+\sum_{q=0}^{Q} x_{2}^{q} \frac{d \varphi_{q}(\xi)}{d \xi} \mathbf{e}_{2} .
\end{gathered}
$$

\section{Straight Boundary Elements}

Let us consider a straight $\mathrm{BE}$ with $Q+1$ nodes situated with equal distance along the element (Fig.1). The global coordinates are given, in term of the local coordinate $\xi \in[-1,1]$ by

$$
x_{1}(\xi)=\sum_{q=0}^{Q} x_{1}^{q} \varphi_{q}(\xi), x_{2}(\xi)=0, n_{1}(\xi)=0, n_{2}(\xi)=1 .
$$

The distance between points $\mathbf{x}$ and $\mathbf{y}$ and the Jacobian in the local coordinates are

$$
r(\xi)=x_{1}(\xi)-y_{1}^{q}, J(\xi)=\sum_{q=0}^{Q} x_{1}^{q} \frac{d \varphi_{q}(\xi)}{d \xi}, \xi \in[-1,1] .
$$

The singular members in (3.5) with (4.6)-(4.8) are reduced to the following form

$$
J_{0}(q)=\int_{-1}^{1} \ln \frac{1}{r} \varphi_{q}(\xi) d \xi, J_{k}(q)=\int_{-1}^{1} \frac{\varphi_{q}(\xi)}{r^{k}} d \xi, k=1,2 .
$$




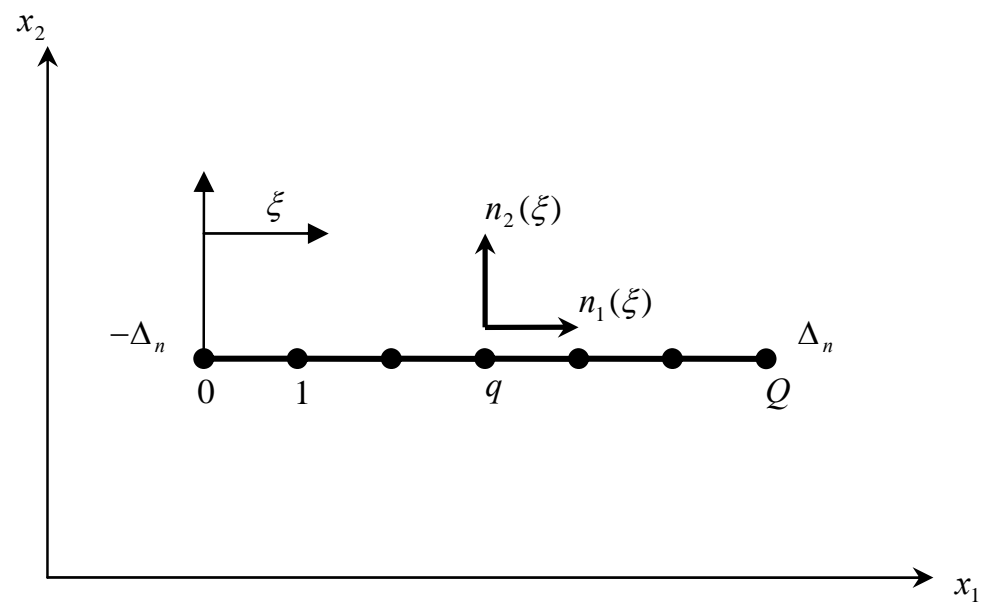

Fig.1

\subsection{Piecewise constant approximation}

The piecewise constant approximation is the simplest one. In this case there is only one node of interpolation and

$$
y_{1}^{0}=0, r=\Delta_{n} \xi .
$$

Interpolation function has the form

$$
\varphi_{0}(\xi)=\left\{\begin{array}{ll}
1 & \forall \xi \in S_{n} \\
0 & \forall \xi \notin S_{n}
\end{array} .\right.
$$

Applying the corresponding formulas from [12] and considering divergent integrals as $(W S),(P V)$ and $(F P)$ we have

$$
\begin{aligned}
J_{0} & =\int_{-1}^{1} \ln \frac{1}{\Delta_{n} \xi} \Delta_{n} d \xi=2 \Delta_{n}\left(1+\ln \left(\frac{1}{\Delta_{n}}\right)\right), \\
J_{1} & =\int_{-1}^{1} \frac{1}{\xi} d \xi=0 \\
J_{2} & =\int_{-1}^{1} \frac{1}{\Delta_{n} \xi^{2}} d \xi=-\frac{2}{\Delta_{n}} .
\end{aligned}
$$




\subsection{Piecewise linear approximation}

There are two nodes of interpolation $q=0$ and $q=1$ with global node coordinates $x_{1}^{0}=-\Delta_{n}, x_{1}^{1}=\Delta_{n}, x_{2}^{0}=0, x_{2}^{1}=0$. The interpolation functions, distance between points $\mathbf{x}$ and $\mathbf{y}$, and the element of the contour length have the form

$$
\varphi_{0}(\xi)=-\frac{\xi-1}{2}, \varphi_{1}(\xi)=\frac{\xi+1}{2}, r=\Delta_{n} \xi-y^{n}, d l=\Delta_{n} d \xi . \xi \in[-1,1] .
$$

The $(W S)$ and $(F P)$ divergent integrals with the kernels (5.3) can be calculated in the same way as for the piecewise constant approximation. The $(P V)$ divergent integrals need a special consideration.

For the node $q=0$ with $y_{1}^{0}=-\Delta_{n}, y_{2}^{0}=0$ and $r=\Delta_{n}(\xi+1)$ we have

$$
\begin{aligned}
& J_{0}(0)=\int_{-1}^{1} \ln \frac{1}{\Delta_{n}(\xi+1)} \frac{1-\xi}{2} \Delta_{n} d \xi=W \cdot S \cdot \int_{0}^{2} \ln \frac{1}{\Delta_{n} \xi} \Delta_{n} d \xi-\int_{0}^{2} \frac{\xi}{2} \ln \frac{1}{\Delta_{n} \xi} \Delta_{n} d \xi= \\
& =\Delta_{n}\left(\frac{3}{2}+\ln \left(\frac{1}{2 \Delta_{n}}\right)\right) \\
& J_{1}(0)=\int_{-1}^{1} \frac{1}{\xi+1} \frac{1-\xi}{2} d \xi=\int_{0}^{2} \frac{2-\xi}{2 \xi} d \xi=P \cdot V \cdot \int_{0}^{2} \frac{1}{\xi} d \xi-\int_{0}^{2} \frac{1}{2} d \xi \rightarrow \infty \\
& J_{2}(0)=\int_{-1}^{1} \frac{1}{\Delta_{n}(\xi+1)^{2}} \frac{1-\xi}{2} d \xi=F \cdot P \cdot \int_{0}^{2} \frac{1}{\Delta_{n} \xi^{2}} d \xi-P \cdot V \cdot \int_{0}^{2} \frac{1}{2 \Delta_{n} \xi} d \xi=-\frac{2}{\Delta_{n}} \\
& J_{0}(1)=\int_{-1}^{1} \ln \frac{1}{\Delta_{n}(\xi+1)} \frac{1+\xi}{2} \Delta_{n} d \xi=\Delta_{n}\left(\frac{1}{2}+\ln \left(\frac{1}{2 \Delta_{n}}\right)\right) \\
& J_{1}(1)=\int_{-1}^{1} \frac{1}{\xi+1} \frac{1+\xi}{2} d \xi=\int_{-1}^{1} \frac{1}{2} d \xi=0, \\
& J_{2}(1)=\int_{-1}^{1} \frac{1}{\Delta_{n}(\xi+1)^{2}} \frac{1+\xi}{2} d \xi=\int_{-1}^{1} \frac{1}{2 \Delta_{n}(\xi+1)} d \xi \rightarrow \infty
\end{aligned}
$$

For the node $q=1$ with $y_{1}^{1}=\Delta_{n}, y_{2}^{1}=0$ and $r=\Delta_{n}(\xi-1)$ we have

$$
\begin{aligned}
& J_{0}(0)=\int_{-1}^{1} \ln \frac{1}{\Delta_{n}(\xi-1)} \frac{\xi-1}{2} \Delta_{n} d \xi=\Delta_{n}\left(\frac{1}{2}+\ln \left(\frac{1}{2 \Delta_{n}}\right)\right), \\
& J_{1}(0)=\int_{-1}^{1} \frac{1}{\xi-1} \frac{\xi-1}{2} d \xi=\int_{-1}^{1} \frac{1}{2} d \xi=0, \\
& J_{2}(0)=\int_{-1}^{1} \frac{1}{\Delta_{n}(\xi-1)^{2}} \frac{\xi-1}{2} d \xi=\int_{-1}^{1} \frac{1}{2 \Delta_{n}(\xi-1)} d \xi \rightarrow \infty,
\end{aligned}
$$




$$
\begin{gathered}
J_{0}(1)=\int_{-1}^{1} \frac{\xi+1}{2} \ln \frac{1}{\Delta_{n}(\xi-1)} \Delta_{n} d \xi=W \cdot S \cdot \int_{-2}^{0} \ln \frac{1}{\Delta_{n} \xi} \Delta_{n} d \xi+\int_{-2}^{0} \frac{\xi}{2} \ln \frac{1}{\Delta_{n} \xi} \Delta_{n} d \xi= \\
=\Delta_{n}\left(\frac{3}{2}+\ln \left(\frac{1}{2 \Delta_{n}}\right)\right), \\
J_{1}(1)=\int_{-1}^{1} \frac{1}{\xi-1} \frac{\xi+1}{2} d \xi=\int_{-2}^{0} \frac{1}{\xi} \frac{\xi+2}{2} d \xi=P \cdot V \cdot \int_{-2}^{0} \frac{1}{\xi} d \xi+\int_{-2}^{0} \frac{1}{2} d \xi \rightarrow-\infty \\
J_{2}(1)=\int_{-1}^{1} \frac{1}{\Delta_{n}(\xi-1)^{2}} \frac{\xi+1}{2} d \xi=F \cdot P \cdot \int_{-2}^{0} \frac{1}{\Delta_{n} \xi^{2}} d \xi+P . V \cdot \int_{-2}^{0} \frac{1}{2 \Delta_{n} \xi} d \xi=-\frac{2}{\Delta_{n}} .
\end{gathered}
$$

Here we have used the following definitions

$$
\text { F.P. } \int_{0}^{1} \frac{1}{x^{2}} d x=\lim _{x \rightarrow 0}\left(\int_{x}^{1} \frac{1}{x^{2}} d x-\frac{1}{x}\right)=-1, W . S . \int_{0}^{1} \ln \frac{1}{x} d x=\lim _{x \rightarrow 0}\left(\int_{x}^{1} \ln \frac{1}{x} d x\right)=1 .
$$

In order to consider the $(P V)$ divergent integrals in the correct way make sure that the Cauchy $(P V)$ integrals are determined for the symmetric interval. Integrals in (5.8b), (5.8f) and (5.9b), (5.9e) are divergent. Situation can be improved if we consider the $n$ - th BE together with the two adjoining the $(n-1)$ and $(n+1)-$ th BEs as shown in Fig. 2.

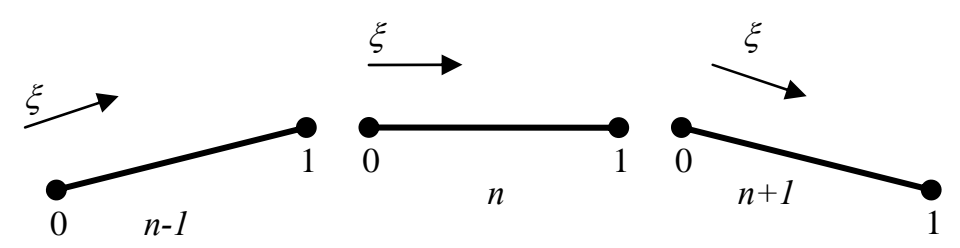

Fig. 2

It is easy to see that together with integral (5.8b) on the $n$-th $\mathrm{BE}$, we can consider integral (5.9b) on the $(n-1)$ - th BE. We have to add these integrals in constructing the global system of BE equations. Thus

$$
J_{1}^{n-1}=\int_{-2}^{0} \frac{1}{\xi} d \xi \rightarrow-\infty, J_{1}^{n}=\int_{0}^{2} \frac{1}{\xi} d \xi \rightarrow \infty, J_{1}^{n-1}+J_{1}^{n}=\int_{-2}^{2} \frac{1}{\xi} d \xi=0 .
$$

In the same way we get

$$
J_{1}^{n}=\int_{-2}^{0} \frac{1}{\xi} d \xi \rightarrow-\infty, J_{1}^{n+1}=\int_{0}^{2} \frac{1}{\xi} d \xi \rightarrow \infty, J_{1}^{n}+J_{1}^{n+1}=\int_{-2}^{2} \frac{1}{\xi} d \xi=0
$$


Since $\int_{-2}^{0} \frac{1}{2} d \xi-\int_{0}^{2} \frac{1}{2} d \xi=0$, we can formally prescribe the value 0 to the $(P V)$ divergent integrals in (5.8b), (5.8f) and (5.9b), (5.9e).

\subsection{Piecewise quadratic approximation}

There are three nodes of interpolation $q=0, q=1$ and $q=2$ with global coordinates $x_{1}^{0}=-\Delta_{n}, x_{1}^{1}=\Delta_{n}, x_{2}^{0}=0, x_{2}^{1}=0$. The interpolation functions, the distance between points $\mathbf{x}$ and $\mathbf{y}$, and the element of the contour length have the form

$$
\begin{gathered}
\varphi_{0}(\xi)=\frac{\xi(\xi-1)}{2}, \varphi_{1}(\xi)=-(\xi-1)(\xi+1), \varphi_{2}(\xi)=\frac{\xi(\xi+1)}{2}, \\
r=\Delta_{n} \xi-y^{n}, d l=\Delta_{n} d \xi .
\end{gathered}
$$

For the node $q=0$ with $y_{1}^{0}=-\Delta_{n}, y_{2}^{0}=0$ and $r=\Delta_{n}(\xi+1)$ we have

$$
\begin{aligned}
& J_{0}(0)=\int_{-1}^{1} \frac{\xi(\xi-1)}{2} \ln \frac{1}{\Delta_{n}(\xi+1)} \Delta_{n} d \xi=\int_{0}^{2} \ln \frac{1}{\Delta_{n} \xi} \Delta_{n} d \xi-\frac{3}{2} \int_{0}^{2} \xi \ln \frac{1}{\Delta_{n} \xi} \Delta_{n} d \xi+\frac{1}{2} \int_{0}^{2} \xi^{2} \ln \frac{1}{\Delta_{n} \xi} \Delta_{n} d \xi= \\
& =\Delta_{n}\left(\frac{17}{18}+\frac{1}{3} \ln \left(\frac{1}{2 \Delta_{n}}\right)\right) \\
& J_{1}(0)=\int_{-1}^{1} \frac{\xi(\xi-1)}{2} \frac{1}{\xi+1} d \xi=\int_{0}^{2} \frac{\xi}{2} d \xi-\int_{0}^{2} \frac{3}{2} d \xi+\int_{0}^{2} \frac{1}{\xi} d \xi=-2 \\
& J_{2}(0)=\int_{-1}^{1} \frac{\xi(\xi-1)}{2} \frac{1}{\Delta_{n}(\xi+1)^{2}} d \xi=\int_{0}^{2} \frac{1}{2 \Delta_{n}} d \xi-\int_{0}^{2} \frac{3}{2} \frac{1}{\Delta_{n} \xi} d \xi+\int_{0}^{2} \frac{1}{\Delta_{n} \xi^{2}} d \xi=-\frac{1}{\Delta_{n}} \\
& J_{0}(1)=\int_{-1}^{1}\left(1-\xi^{2}\right) \ln \frac{1}{\Delta_{n}(\xi+1)} \Delta_{n} d \xi=2 \int_{0}^{2} \xi \ln \frac{1}{\Delta_{n} \xi} \Delta_{n} d \xi-\int_{0}^{2} \xi^{2} \ln \frac{1}{\Delta_{n} \xi} \Delta_{n} d \xi= \\
& J_{1}(1)=\int_{-1}^{1}\left(1-\xi^{2}\right) \frac{1}{\xi+1} d \xi=\int_{-1}^{1}(1-\xi) d \xi=2 \\
& J_{2}(1)=\int_{-1}^{1}\left(1-\xi^{2}\right) \frac{1}{\Delta_{n}(\xi+1)^{2}} d \xi=\int_{0}^{2} \frac{2}{\Delta_{n} \xi} d \xi-\int_{0}^{2} \frac{1}{\Delta_{n}} d \xi=-\frac{2}{\Delta_{n}} \\
& J_{0}(2)=\int_{-1}^{1} \frac{\xi(\xi+1)}{2} \ln \frac{1}{\Delta_{n}(\xi+1)} \Delta_{n} d \xi=-\frac{1}{2} \int_{0}^{2} \xi \ln \frac{1}{\Delta_{n} \xi} \Delta_{n} d \xi+\frac{1}{2} \int_{0}^{2} \xi^{2} \ln \frac{1}{\Delta_{n} \xi} \Delta_{n} d \xi=
\end{aligned}
$$




$$
\begin{aligned}
& J_{1}(2)=\int_{-1}^{1} \frac{\xi(\xi+1)}{2} \frac{1}{\xi+1} d \xi=\int_{-1}^{1} \frac{\xi}{2} d \xi=0, \\
& J_{2}(2)=\int_{-1}^{1} \frac{\xi(\xi+1)}{2} \frac{1}{\Delta_{n}(\xi+1)^{2}} d \xi=\int_{0}^{2} \frac{1}{2 \Delta_{n}} d \xi-\int_{0}^{2} \frac{1}{2 \Delta_{n} \xi^{2}} d \xi=\frac{1}{\Delta_{n}} .
\end{aligned}
$$

For the node $q=1$ with $y_{1}^{1}=0, y_{2}^{1}=0$ and $r=\Delta_{n} \xi$ we have

$$
\begin{aligned}
& J_{0}(0)=\int_{-1}^{1} \frac{\xi(\xi-1)}{2} \ln \frac{1}{\Delta_{n} \xi} \Delta_{n} d \xi=\frac{1}{9} \Delta_{n}\left(1-3 \ln \left(\frac{1}{2 \Delta_{n}}\right)\right), \\
& J_{1}(0)=\int_{-1}^{1} \frac{1}{\xi} \frac{\xi(\xi-1)}{2} d \xi=\int_{-1}^{1} \frac{\xi-1}{2} d \xi=-1, \\
& J_{2}(0)=\int_{-1}^{1} \frac{1}{\Delta_{n} \xi^{2}} \frac{\xi(\xi-1)}{2} d \xi=\int_{-1}^{1} \frac{1}{2 \Delta_{n}} d \xi-\int_{-1}^{1} \frac{1}{2 \Delta_{n} \xi} d \xi=\frac{1 \mid}{\Delta_{n}}, \\
& J_{0}(1)=\int_{-1}^{1} \ln \frac{1}{\Delta_{n} \xi}\left(1-\xi^{2}\right) \Delta_{n} d \xi=\frac{4}{9} \Delta_{n}\left(4-3 \ln \left(\frac{1}{2 \Delta_{n}}\right)\right), \\
& J_{1}(1)=\int_{-1}^{1} \frac{1}{\xi}\left(1-\xi^{2}\right) d \xi=P \cdot V \cdot \int_{-1}^{1} \frac{1}{\xi} d \xi-\int_{-1}^{1} \xi d \xi=0, \\
& J_{2}(1)=\int_{-1}^{1} \frac{1}{\Delta_{n} \xi^{2}}\left(1-\xi^{2}\right) d \xi=\int_{-1}^{1} \frac{1}{\Delta_{n} \xi^{2}} d \xi-\int_{-1}^{1} \frac{1}{\Delta_{n}} d \xi=-\frac{4}{\Delta_{n}}, \\
& J_{0}(2)=\int_{-1}^{1} \frac{\xi(\xi-1)}{2} \ln \frac{1}{\Delta_{n} \xi} \Delta_{n} d \xi=\frac{1}{9} \Delta_{n}\left(1-3 \ln \left(\frac{1}{2 \Delta_{n}}\right)\right), \\
& J_{1}(2)=\int_{-1}^{1} \frac{1}{\xi} \frac{\xi(\xi+1)}{2} d \xi=\int_{-1}^{1} \frac{\xi+1}{2} d \xi=1, \\
& J_{2}(2)=\int_{-1}^{1} \frac{1}{\Delta_{n} \xi^{2}} \frac{\xi(\xi+1)}{2} d \xi=\int_{-1}^{1} \frac{1}{2 \Delta_{n}} d \xi+\int_{-1}^{1} \frac{1}{2 \Delta_{n} \xi} d \xi=\frac{1}{\Delta_{n}} .
\end{aligned}
$$

For the node $q=2$ with $y_{1}^{2}=\Delta_{n}, y_{2}^{2}=0$ and $r=\Delta_{n}(\xi-1)$ we have

$$
\begin{aligned}
& J_{0}(0)=\int_{-1}^{1} \frac{\xi(\xi-1)}{2} \ln \frac{1}{\Delta_{n}(\xi-1)} \Delta_{n} d \xi=\frac{1}{2} \int_{-2}^{0} \xi \ln \frac{1}{\Delta_{n} \xi} \Delta_{n} d \xi+\frac{1}{2} \int_{-2}^{0} \xi^{2} \ln \frac{1}{\Delta_{n} \xi} \Delta_{n} d \xi= \\
& =\Delta_{n}\left(\frac{17}{18}+\frac{1}{3} \ln \left(\frac{1}{2 \Delta_{n}}\right)\right), \\
& J_{1}(0)=\int_{-1}^{1} \frac{\xi(\xi-1)}{2} \frac{1}{\xi-1} d \xi=\int_{-2}^{0} \frac{\xi}{2} d \xi=0,
\end{aligned}
$$




$$
\begin{aligned}
& J_{2}(0)=\int_{-1}^{1} \frac{\xi(\xi-1)}{2} \frac{1}{\Delta_{n}(\xi-1)^{2}} d \xi=\frac{1}{2} \int_{-2}^{0} \frac{1}{\Delta_{n}} d \xi+\frac{1}{2} \int_{-2}^{0} \frac{1}{\Delta_{n} \xi} d \xi=\frac{1}{\Delta_{n}}, \\
& J_{0}(1)=\int_{-1}^{1}\left(1-\xi^{2}\right) \ln \frac{1}{\Delta_{n}(\xi-1)} \Delta_{n} d \xi=-2 \int_{-2}^{0} \xi \ln \frac{1}{\Delta_{n} \xi} \Delta_{n} d \xi-\int_{-2}^{0} \xi^{2} \ln \frac{1}{\Delta_{n} \xi} \Delta_{n} d \xi= \\
& =2 \Delta_{n}\left(\frac{5}{9}+\frac{2}{3} \ln \left(\frac{1}{2 \Delta_{n}}\right)\right) \text {, } \\
& J_{1}(1)=\int_{-1}^{1}\left(1-\xi^{2}\right) \frac{1}{\xi+1} d \xi=\int_{-1}^{1}(1-\xi) d \xi=2, \\
& J_{2}(1)=\int_{-1}^{1}\left(1-\xi^{2}\right) \frac{1}{\Delta_{n}(\xi-1)^{2}} d \xi=-\int_{-2}^{0} \frac{2}{\Delta_{n} \xi} d \xi-\int_{-2}^{0} \frac{1}{\Delta_{n}} d \xi=-\frac{2}{\Delta_{n}} \text {, } \\
& J_{0}(2)=\int_{-1}^{1} \frac{\xi(\xi+1)}{2} \ln \frac{1}{\Delta_{n}(\xi-1)} \Delta_{n} d \xi=\int_{-2}^{0} \ln \frac{1}{\Delta_{n} \xi} \Delta_{n} d \xi+\frac{3}{2} \int_{-2}^{0} \xi \ln \frac{1}{\Delta_{n} \xi} \Delta_{n} d \xi+\frac{1}{2} \int_{-2}^{0} \xi^{2} \ln \frac{1}{\Delta_{n} \xi} \Delta_{n} d \xi= \\
& =\Delta_{n}\left(\frac{17}{18}+\frac{1}{3} \ln \left(\frac{1}{2 \Delta_{n}}\right)\right) \text {, } \\
& J_{1}(2)=\int_{-1}^{1} \frac{\xi(\xi+1)}{2} \frac{1}{\xi-1} d \xi=\int_{-2}^{0} \frac{\xi}{2} d \xi+\int_{-2}^{0} \frac{3}{2} d \xi+\int_{-2}^{0} \frac{1}{\xi} d \xi \rightarrow 2, \\
& J_{2}(2)=\int_{-1}^{1} \frac{\xi(\xi+1)}{2} \frac{1}{\Delta_{n}(\xi-1)^{2}} d \xi=\int_{-2}^{0} \frac{1}{2 \Delta_{n}} d \xi+\int_{-2}^{0} \frac{3}{2} \frac{1}{\Delta_{n} \xi} d \xi+\int_{-2}^{0} \frac{1}{\Delta_{n} \xi^{2}} d \xi=-\frac{1}{\Delta_{n}} .
\end{aligned}
$$

The singular integrals at the end nodes have to be calculated in the same way as for the piecewise linear approximation.

\section{Curved Boundary Elements}

Now we try to calculate divergent integrals from (3.5) for the general curved BEs. Unfortunately, in the case of the curved BEs the regularization formulas from $[11,12]$ can not be applied directly. Therefore we transform these integrals in the following way

$$
\begin{aligned}
& \int_{\partial V_{n}} U_{i j}\left(\mathbf{x}, \mathbf{y}^{q}\right) \varphi_{q}(\mathbf{x}) d S=\int_{\partial V_{n}}\left\{U_{i j}\left(\mathbf{x}, \mathbf{y}^{q}\right) \varphi_{q}(\mathbf{x})-U_{i j}\left[\pi(\mathbf{x}), \mathbf{y}^{q}\right]\right\} d S+\int_{\Pi_{n}} U_{i j}\left[\pi(\mathbf{x}), \mathbf{y}^{q}\right] d S_{\pi}, \\
& \int_{\partial V_{n}} W_{i j}\left(\mathbf{x}, \mathbf{y}^{q}\right) \varphi_{q}(\mathbf{x}) d S=\int_{\partial V_{n}}\left\{W_{i j}\left(\mathbf{x}, \mathbf{y}^{q}\right) \varphi_{q}(\mathbf{x})-W_{i j}\left[\pi(\mathbf{x}), \mathbf{y}^{q}\right]\right\} d S+\int_{\Pi_{n}} W_{i j}\left[\pi(\mathbf{x}), \mathbf{y}^{q}\right] d S_{\pi}, \\
& \int_{\partial V_{n}} K_{i j}\left(\mathbf{x}, \mathbf{y}^{q}\right) \varphi_{q}(\mathbf{x}) d S=\int_{\partial V_{n}}\left\{K_{i j}\left(\mathbf{x}, \mathbf{y}^{q}\right) \varphi_{q}(\mathbf{x})-K_{i j}\left[\pi(\mathbf{x}), \mathbf{y}^{q}\right]\right\} d S+\int_{\Pi_{n}} K_{i j}\left[\pi(\mathbf{x}), \mathbf{y}^{q}\right] d S_{\pi}, \\
& \int_{\partial V_{n}} F_{i j}\left(\mathbf{x}, \mathbf{y}^{q}\right) \varphi_{q}(\mathbf{x}) d S=\int_{\partial V_{n}}\left\{F_{i j}\left(\mathbf{x}, \mathbf{y}^{q}\right) \varphi_{q}(\mathbf{x})-F_{i j}\left[\pi(\mathbf{x}), \mathbf{y}^{q}\right]\right\} d S+\int_{\Pi_{n}} F_{i j}\left[\pi(\mathbf{x}), \mathbf{y}^{q}\right] d S_{\pi},
\end{aligned}
$$


where $\pi: \partial V_{n} \rightarrow \Pi_{n}$ is the operator of an orthogonal projection from the curved element $\partial V_{n}$ to the linear element $\Pi_{\varepsilon}, d S_{\pi}$ is the differential element of length for a linear element $\Pi_{\varepsilon}$. The main idea of these transformations is that the divergent integral over curved element is presented as a sum of the regular integral over the curved element and the divergent integral over the flat element. As shown before, the kernels in (4.6)-(4.8) have the simple form (5.3) for the straight BE and can be calculated analytically. We will illustrate here this approach with some divergent integrals.

\subsection{Piecewise quadratic approximation}

In the case of the piecewise quadratic approximation there are three nodes of interpolation $q=0, q=1$ and $q=2$. Interpolation functions and their derivatives have the form

$$
\begin{gathered}
\varphi_{0}(\xi)=\frac{\xi(\xi-1)}{2}, \varphi_{1}(\xi)=-(\xi-1)(\xi+1), \varphi_{2}(\xi)=\frac{\xi(\xi+1)}{2}, \\
\frac{d \varphi_{0}(\xi)}{d \xi}=\xi-\frac{1}{2}, \frac{d \varphi_{1}(\xi)}{d \xi}=-2 \xi, \frac{d \varphi_{2}(\xi)}{d \xi}=\xi+\frac{1}{2} .
\end{gathered}
$$

The global coordinates as functions of the local coordinate are

$$
x_{i}(\xi)=\sum_{q=0}^{2} x_{i}^{q} \varphi_{q}(\xi), y_{i}(\zeta)=\sum_{q=0}^{2} y_{i}^{q} \varphi_{q}(\zeta) .
$$

For convenience, we present them in the form

$$
x_{i}=a_{i} \xi^{2}+b_{i} \xi+x_{i}^{1}, a_{i}=\frac{\left(x_{i}^{0}-2 x_{i}^{1}+x_{i}^{2}\right)}{2}, b_{i}=\frac{\left(x_{i}^{2}-x_{i}^{0}\right)}{2},
$$

where $x_{i}^{0}, x_{i}^{1}$ and $x_{i}^{2}$ are global coordinates of nodal points of the BE.

The curved element is

$$
d S=J(\xi) d \xi
$$

where Jacobian has the form

$$
J(\xi)=\sqrt{\left(\sum_{q=0}^{2} x_{1}^{q} \frac{d \varphi_{q}(\xi)}{d \xi}\right)^{2}+\left(\sum_{q=0}^{2} x_{2}^{q} \frac{d \varphi_{q}(\xi)}{d \xi}\right)^{2}}=\sqrt{\left(2 a_{1} \xi+b_{1}\right)^{2}+\left(2 a_{2} \xi+b_{2}\right)^{2}} .
$$

The components of the normal vector in the local coordinates are

$$
n_{1}=\frac{1}{J(\xi)} \frac{d x_{2}}{d \xi}=\frac{\left(2 a_{2} \xi+b_{2}\right)}{\sqrt{\left(a_{1} \xi+b_{1}\right)^{2}+\left(a_{2} \xi+b_{2}\right)^{2}}},
$$




$$
n_{2}=\frac{-1}{J(\xi)} \frac{d x_{1}}{d \xi}=-\frac{\left(2 a_{1} \xi+b_{1}\right)}{\sqrt{\left(a_{1} \xi+b_{1}\right)^{2}+\left(a_{2} \xi+b_{2}\right)^{2}}} .
$$

Distance between the nodal point $\mathbf{y}^{q}$ and current point $\mathbf{x}$ is

$$
r\left(\xi, \mathbf{y}^{q}\right)=\sqrt{\left(a_{1} \xi^{2}+b_{1} \xi+x_{1}^{1}-y_{1}^{q}\right)^{2}+\left(a_{2} \xi^{2}+b_{2} \xi+x_{2}^{1}-y_{2}^{q}\right)^{2}} d \xi .
$$

Now we will illustrate the developed method of the regularization for the divergent integrals in the case of the curved BE. On each BE there are three nodal points and three interpolation polynomials. It means that nine integral have to be calculated. Obviously these integrals are divergent only in the case when the nodal point and the interpolation polynomial have the same index $q$. Bellow, we consider each integral in detail and present corresponding regularization formulas.

The weakly singular integrals related to the fundamental solutions from (4.8) have the from

$$
J_{0}(q)=\int_{-1}^{1} \ln \frac{1}{r\left(\xi, \mathbf{y}^{0}\right)} \varphi_{q}(\xi) J(\xi) d \xi
$$

The regularized equations have the following form.

For node $q=0$, where $y_{1}^{0}=x_{1}^{0}$

$$
\begin{aligned}
J_{0}(0)=\int_{-1}^{1}\left(\ln \frac{1}{r\left(\xi, \mathbf{y}^{0}\right)} \varphi_{0}(\xi) J(\xi)-S^{0,1} \ln \frac{1}{\xi+1}\right) d \xi+W . S \cdot \int_{-1}^{1} S^{0,1} \ln \frac{1}{\xi+1} d \xi \\
J_{0}(1)=\int_{-1}^{1} \ln \frac{1}{r\left(\xi, \mathbf{y}^{0}\right)} \varphi_{1}(\xi) J(\xi) d \xi \\
J_{0}(2)=\int_{-1}^{1} \ln \frac{1}{r\left(\xi, \mathbf{y}^{0}\right)} \varphi_{2}(\xi) J(\xi) d \xi
\end{aligned}
$$

It is easy to show that only the second integral in (6.10a) is divergent. In order to proof that, we will consider limits of the expressions inside the correspondent integrals at the nodal point. The equations below show that these expressions have finite values

$$
\begin{gathered}
L_{0}=\lim _{\xi \rightarrow-1}\left(\ln \frac{1}{r\left(\xi, \mathbf{y}^{0}\right)} \varphi_{0}(\xi) J(\xi)-S^{0,1} \ln \frac{1}{\xi+1}\right)=-\frac{1}{2} S^{0,1} \ln \left(\frac{1}{4 \sqrt{\left(l_{1}^{0,2}\right)^{2}+\left(l_{2}^{0,2}\right)^{2}}}\right), \\
L_{1}=\lim _{\xi \rightarrow-1} \varphi_{1}(\xi) J(\xi) \ln \frac{1}{r\left(\xi, \mathbf{y}^{0}\right)}=0, \quad L_{2}=\lim _{\xi \rightarrow-1} \varphi_{2}(\xi) J(\xi) \ln \frac{1}{r\left(\xi, \mathbf{y}^{0}\right)}=0 .
\end{gathered}
$$

Here and below the following notation have been used

$$
S^{\alpha, \beta}=\sqrt{\left(x_{1}^{\alpha}-x_{1}^{\beta}\right)^{2}+\left(x_{2}^{\alpha}-x_{2}^{\beta}\right)^{2}}, \quad l_{i}^{\alpha, \beta}=3 x_{1}^{\alpha}-4 x_{1}^{1}+x_{1}^{\beta} .
$$


The WS integral in (6.10a) can be easy calculated using equation (5.6a) and definition (5.10).

For node $q=1$, where $y_{1}^{1}=x_{1}^{1}$

$$
\begin{gathered}
J_{0}(0)=\int_{-1}^{1} \ln \frac{1}{r\left(\xi, \mathbf{y}^{1}\right)} \varphi_{0} J(\xi) d \xi, \\
J_{0}(1)=\int_{-1}^{1}\left(\ln \frac{1}{r\left(\xi, \mathbf{y}^{1}\right)} \varphi_{0}(\xi) J(\xi)-S^{0,2} \ln \frac{1}{\xi}\right) d \xi+W . S . \int_{-1}^{1} S^{0,2} \ln \left|\frac{1}{\xi}\right| d \xi \\
J_{0}(2)=\int_{-1}^{1} \ln \frac{1}{r\left(\xi, \mathbf{y}^{1}\right)} \varphi_{1} J(\xi) d \xi .
\end{gathered}
$$

Here also only the second integral in (6.11b) is divergent. The following equations show that

$$
\begin{aligned}
& L_{1}=\lim _{\xi \rightarrow 0}\left(\ln \frac{1}{r\left(\xi, \mathbf{y}^{1}\right)} \varphi_{0}(\xi) J(\xi)-S^{0,2} \ln \left|\frac{1}{\xi}\right|\right)=-\frac{1}{2} S^{0,2}\left(\ln (2)-\ln \left(S^{0,2}\right)\right), \\
& L_{0}=\lim _{\xi \rightarrow 0} \varphi_{0}(\xi) J(\xi) \ln \frac{1}{r\left(\xi, \mathbf{y}^{1}\right)}=0, \quad L_{2}=\lim _{\xi \rightarrow 0} \varphi_{2}(\xi) J(\xi) \ln \frac{1}{r\left(\xi, \mathbf{y}^{1}\right)}=0 .
\end{aligned}
$$

The $W S$ integral in (6.11b) can be easy calculated using equation (5.6a).

For node $q=2$, where $y_{1}^{2}=x_{1}^{2}$

$$
\begin{gathered}
J_{0}(0)=\int_{-1}^{1} \ln \frac{1}{r\left(\xi, \mathbf{y}^{2}\right)} \varphi_{0}(\xi) J(\xi) d \xi \\
J_{0}(1)=\int_{-1}^{1} \ln \frac{\xi-1}{r\left(\xi, \mathbf{y}^{2}\right)} \varphi_{1}(\xi) J(\xi) d \xi \\
J_{0}(2)=\int_{-1}^{1}\left(\ln \frac{1}{r\left(\xi, \mathbf{y}^{2}\right)} \varphi_{2}(\xi) J(\xi)-S^{1,2} \ln \frac{1}{\xi-1}\right) d \xi+W \cdot S \cdot \int_{-1}^{1} S^{1,2} \ln \frac{1}{\xi-1} d \xi .
\end{gathered}
$$

Here we have the same situation as in the case of $q=0$, only second integral in (6.12c) is divergent. The following equations show that

$$
\begin{gathered}
L_{2}=\lim _{\xi \rightarrow 1}\left(\ln \frac{1}{r\left(\xi, \mathbf{y}^{2}\right)} \varphi_{2}(\xi) J(\xi)-S^{1,2} \ln \frac{1}{\xi+1}\right)=-\frac{1}{2} S^{1,2} \ln \left(\frac{1}{4 \sqrt{\left(l_{1}^{0,2}\right)^{2}+\left(l_{2}^{0,2}\right)^{2}}}\right), \\
L_{0}=\lim _{\xi \rightarrow 1} \varphi_{0}(\xi) J(\xi) \ln \frac{1}{r\left(\xi, \mathbf{y}^{2}\right)}=0, L_{1}=\lim _{\xi \rightarrow 1} \varphi_{1}(\xi) J(\xi) \ln \frac{1}{r\left(\xi, \mathbf{y}^{2}\right)}=0 .
\end{gathered}
$$

The WS integral in (6.11b) can be easy calculated using equation (5.6a) and definition (5.10). 
Next, we consider the singular integral of the form

$$
J_{1}=\int_{-1}^{1} \frac{1}{r\left(\xi, \mathbf{y}^{q}\right)} \varphi_{q}(\xi) J(\xi) d \xi .
$$

The regularized equations have the following form.

For node $q=0$, where $y_{1}^{0}=x_{1}^{0}$ we have to calculate

$$
\begin{gathered}
J_{1}(0)=\int_{-1}^{1} \frac{\varphi_{0}(\xi) J(\xi)(\xi+1)-L_{0} r\left(\xi, \mathbf{y}^{0}\right)^{2}}{r\left(\xi, \mathbf{y}^{0}\right)(\xi+1)} d \xi+P . V \cdot \int_{-1}^{1} \frac{L_{0}}{(\xi+1)} d \xi, \\
J_{1}(1)=\int_{-1}^{1} \frac{\varphi_{1}(\xi) J(\xi)}{r\left(\xi, \mathbf{y}^{0}\right)} d \xi, \\
J_{1}(2)=\int_{-1}^{1} \frac{\varphi_{2}(\xi) J(\xi)}{r\left(\xi, \mathbf{y}^{0}\right)} d \xi .
\end{gathered}
$$

The following equations show that only second integral in (6.14a) is divergent.

$$
\begin{gathered}
L_{0}=\lim _{\xi \rightarrow-1} \frac{\varphi_{0}(\xi) J(\xi)(\xi+1)}{r\left(\xi, \mathbf{y}^{0}\right)}=\frac{2 S^{0,1}}{\sqrt{\left(l_{1}^{0,2}\right)^{2}+\left(l_{2}^{0,2}\right)^{2}}}, \\
L_{1}=\lim _{\xi \rightarrow-1} \frac{\varphi_{1}(\xi) J(\xi)}{r\left(\xi, \mathbf{y}^{0}\right)}=0, \quad L_{2}=\lim _{\xi \rightarrow-1} \frac{\varphi_{2}(\xi) J(\xi)}{r\left(\xi, \mathbf{y}^{0}\right)}=0 .
\end{gathered}
$$

The $P V$ integral in (6.14a) can be easy calculated using equation (5.6b) and definition (5.11a).

For node $q=1$, where $y_{1}^{1}=x_{1}^{1}$

$$
\begin{gathered}
J_{1}(0)=\int_{-1}^{1} \frac{\varphi_{0}(\xi) J(\xi)}{r\left(\xi, \mathbf{y}^{1}\right)} d \xi, \\
J_{1}(0)=\int_{-1}^{1} \frac{\varphi_{0}(\xi) J(\xi) \xi-r\left(\xi, \mathbf{y}^{1}\right)^{2}}{r\left(\xi, \mathbf{y}^{1}\right) \xi} d \xi+P . V \cdot \int_{-1}^{1} \frac{1}{\xi} d \xi, \\
J_{1}(2)=\int_{-1}^{1} \frac{\varphi_{2}(\xi) J(\xi)}{r\left(\xi, \mathbf{y}^{1}\right)} d \xi .
\end{gathered}
$$

Limit calculation shows that only second integral in (6.15b) is divergent

$$
L_{0}=\lim _{\xi \rightarrow 0} \frac{\varphi_{0}(\xi) J(\xi)}{r\left(\xi, \mathbf{y}^{1}\right)}=0, \quad L_{1}=\lim _{\xi \rightarrow-1} \frac{\varphi_{1}(\xi) J(\xi) \xi}{r\left(\xi, \mathbf{y}^{1}\right)}=1, \quad L_{2}=\lim _{\xi \rightarrow 0} \frac{\varphi_{2}(\xi) J(\xi)}{r\left(\xi, \mathbf{y}^{1}\right)}=0 .
$$

The $P V$ integral in (6.15b) can be easy calculated using equation (5.6b).

For node $q=2$, where $y_{1}^{2}=x_{1}^{2}$ 


$$
\begin{gathered}
J_{1}(0)=\int_{-1}^{1} \frac{\varphi_{0}(\xi) J(\xi)}{r\left(\xi, \mathbf{y}^{2}\right)} d \xi, \\
J_{1}(1)=\int_{-1}^{1} \frac{\varphi_{1}(\xi) J(\xi)}{r\left(\xi, \mathbf{y}^{2}\right)} d \xi, \\
J_{1}(2)=\int_{-1}^{1} \frac{\varphi_{2}(\xi) J(\xi)(\xi-1)-L_{2} r\left(\xi, \mathbf{y}^{2}\right)^{2}}{r\left(\xi, \mathbf{y}^{2}\right)^{2}(\xi-1)} d \xi+P . V \cdot \int_{-1}^{1} \frac{L_{2}}{(\xi-1)} d \xi .
\end{gathered}
$$

Here we have the same situation as in the case of $q=0$. The following equations show that only second integral in (6.16c) is divergent

$$
\begin{gathered}
L_{2}=\lim _{\xi \rightarrow 1} \frac{\varphi_{2}(\xi) J(\xi)(\xi-1)}{r\left(\xi, \mathbf{y}^{2}\right)}=\frac{2 S^{1,2}}{\sqrt{\left(l_{1}^{0,2}\right)^{2}+\left(l_{2}^{0,2}\right)^{2}}}, \\
L_{0}=\lim _{\xi \rightarrow 1} \ln \frac{\varphi_{0}(\xi) J(\xi)}{r\left(\xi, \mathbf{y}^{2}\right)}=0, L_{1}=\lim _{\xi \rightarrow 1} \frac{\varphi_{2}(\xi) J(\xi)}{r\left(\xi, \mathbf{y}^{2}\right)}=0 .
\end{gathered}
$$

The $P V$ integral in (6.16c) can be easy calculated using equation (5.6b) and definition (5.11a).

Finally let us consider hypersingular integral

$$
J_{2}=\int_{-1}^{1} \frac{1}{r\left(\xi, \mathbf{y}^{q}\right)^{2}} \varphi_{q}(\xi) J(\xi) d \xi .
$$

The regularized equations have the following form.

For node $q=0$, where $y_{1}^{0}=x_{1}^{0}$ we have to calculate

$$
\begin{gathered}
J_{2}(0)=\int_{-1}^{1} \frac{\varphi_{0}(\xi) J(\xi)(\xi+1)^{2}-L_{0} r\left(\xi, \mathbf{y}^{0}\right)^{2}}{r\left(\xi, \mathbf{y}^{0}\right)^{2}(\xi+1)^{2}} d \xi+F \cdot P \cdot \int_{-1}^{1} \frac{L_{0}}{(\xi+1)^{2}} d \xi, \\
J_{2}(1)=\int_{-1}^{1} \frac{\varphi_{2}(\xi) J(\xi)}{r\left(\xi, \mathbf{y}^{0}\right)^{2}} d \xi \\
J_{2}(2)=\int_{-1}^{1} \frac{\varphi_{2}(\xi) J(\xi)}{r\left(\xi, \mathbf{y}^{0}\right)^{2}} d \xi .
\end{gathered}
$$

The following limit calculation shows that only second integral in (6.18a) is divergent

$$
\begin{gathered}
L_{0}=\lim _{\xi \rightarrow-1} \frac{\varphi_{0}(\xi) J(\xi)(\xi+1)^{2}}{r\left(\xi, \mathbf{y}^{0}\right)^{2}}=\frac{4 S^{0,1}}{\left(l_{1}^{0,2}\right)^{2}+\left(l_{2}^{0,2}\right)^{2}}, \\
L_{1}=\lim _{\xi \rightarrow-1} \frac{\varphi_{1}(\xi) J(\xi)}{r\left(\xi, \mathbf{y}^{0}\right)^{2}}=0, \quad L_{2}=\lim _{\xi \rightarrow-1} \frac{\varphi_{2}(\xi) J(\xi)}{r\left(\xi, \mathbf{y}^{0}\right)^{2}}=0 .
\end{gathered}
$$

The FP integral in (6.18a) can be easy calculated using equation (5.6c) and definition (5.10). 
For node $q=1$, where $y_{1}^{1}=x_{1}^{1}$

$$
\begin{gathered}
J_{2}(1)=\int_{-1}^{1} \frac{\varphi_{2}(\xi) J(\xi)}{r\left(\xi, \mathbf{y}^{1}\right)^{2}} d \xi, \\
J_{2}(1)=\int_{-1}^{1} \frac{\varphi_{1}(\xi) J(\xi) \xi^{2}-L_{1} r\left(\xi, \mathbf{y}^{1}\right)^{2}}{r\left(\xi, \mathbf{y}^{1}\right)^{2} \xi^{2}} d \xi+F \cdot P \cdot \int_{-1}^{1} \frac{L_{1}}{\xi^{2}} d \xi, \\
J_{2}(2)=\int_{-1}^{1} \frac{\varphi_{2}(\xi) J(\xi)}{r\left(\xi, \mathbf{y}^{1}\right)^{2}} d \xi .
\end{gathered}
$$

The following equations show that only second integral in (6.18b) is divergent

$$
L_{0}=\lim _{\xi \rightarrow 0} \frac{\varphi_{0}(\xi) J(\xi)}{r\left(\xi, \mathbf{y}^{1}\right)^{2}}=0, L_{1}=\lim _{\xi \rightarrow 0} \frac{\varphi_{1}(\xi) J(\xi) \xi}{r\left(\xi, \mathbf{y}^{1}\right)^{2}}=\frac{2}{\left(S^{0,2}\right)^{2}}, \quad L_{2}=\lim _{\xi \rightarrow 0} \frac{\varphi_{2}(\xi) J(\xi)}{r\left(\xi, \mathbf{y}^{1}\right)^{2}}=0 .
$$

The $F P$ integral in (6.19b) can be easy calculated using equation (5.6c) and definition (5.10).

For node $q=2$, where $y_{1}^{2}=x_{1}^{2}$

$$
\begin{gathered}
J_{2}(0)=\int_{-1}^{1} \frac{\varphi_{2}(\xi) J(\xi)}{r\left(\xi, \mathbf{y}^{2}\right)^{2}} d \xi, \\
J_{2}(1)=\int_{-1}^{1} \frac{\varphi_{2}(\xi) J(\xi)}{r\left(\xi, \mathbf{y}^{2}\right)^{2}} d \xi, \\
J_{2}(2)=\int_{-1}^{1} \frac{\varphi_{2}(\xi) J(\xi)(\xi-1)^{2}-L_{2} r\left(\xi, \mathbf{y}^{0}\right)^{2}}{r\left(\xi, \mathbf{y}^{0}\right)^{2} \xi^{2}}+F \cdot P \cdot \int_{-1}^{1} \frac{L_{2}}{(\xi-1)^{2}} d \xi .
\end{gathered}
$$

Here we have the same situation as in the case of $q=0$. Limit calculation shows that only second integral in (6.20c) is divergent

$$
\begin{gathered}
L_{2}=\lim _{\xi \rightarrow 1} \frac{\varphi_{2}(\xi) J(\xi)(\xi-1)^{2}}{r\left(\xi, \mathbf{y}^{2}\right)^{2}}=\frac{4 S^{1,2}}{\left(l_{1}^{2,0}\right)^{2}+\left(l_{2}^{2,0}\right)^{2}}, \\
L_{0}=\lim _{\xi \rightarrow 1} \ln \frac{\varphi_{0}(\xi) J(\xi)}{r\left(\xi, \mathbf{y}^{2}\right)^{2}}=0, \quad L_{1}=\lim _{\xi \rightarrow 1} \frac{\varphi_{2}(\xi) J(\xi)}{r\left(\xi, \mathbf{y}^{2}\right)^{2}}=0 .
\end{gathered}
$$

The $F P$ integral in (6.19b) can be easy calculated using equation (5.6c) and definition (5.10).

Many divergent integrals of various types appear in the equations (4.6)-(4.8). Since methodology of their regularization is the same as it was presented above, only their regularized equations are presented in Appendixes A-C. 


\section{Conclusions}

Method of the divergent integral regularization, which is based on the theory of distribution is developed here and is applied to the BIE solution of the 2-D elastostatic problems. The weakly singular, singular and hypersingular integrals in 1-D are considered and their regular formulas have been obtained using the unified technique. Straight and curved BEs with different number of nodal points have been considered. In the case of the straight BE, all integrals can be calculated analytically and in the case of curvilinear $\mathrm{BE}$, numerical integration of regular parts of the corresponding divergent integrals has to be used.

\section{References}

[1] Becker A.A. The boundary element method in engineering. A complete course. McGraw-Hill, New York , 1992

[2] Brebbia C.A., Dominguez J. Boundary elements. An introductory course, WIT Press, Southampton, 1998.

[3] Chen G. and Zhou J. Boundary Element Method, Academic Press, London 1992, $646 \mathrm{p}$

[4] Chen J.T. and Hong H.-K. Review of dual boundary element methods with emphasis on hypersingular integrals and divergent series. Applied Mechanics Review, 52(1), pp. 17-33, 1999.

[5] Guz A.N. and Zozulya V.V. Fracture dynamics with allowance for a crack edges contact interaction, International Journal of Nonlinear Sciences and Numerical Simulation, 2(3), pp. 173-233, 2001.

[6] Guz A.N. and Zozulya V.V. Elastodynamic unilateral contact problems with friction for bodies with cracks, International Applied Mechanics, 38(8), pp. 3-45, 2002.

[7] Sladek V. and Sladek J. (eds.) Singular Integrals in Boundary Element Methods, WIT Press, Southampton, 1998.

[8] Tanaka, M., Sladek, V. and Sladek, J. Regularization techniques applied to boundary element methods. Applied Mechanics Reviews, 47(10) (1994) 457-499

[9] Zienkiewicz O.C., Morgan K. Finite elements and approximation. John Wiley \& Sons, New York, 1983

[10] Zozulya V.V., Integrals of Hadamard type in dynamic problem of the crack theory. Doklady Academii Nauk. UkrSSR, Ser. A. Physical Mathematical \& Technical Sciences, 2, pp.19-22, 1991, (in Russian).

[11] Zozulya V.V. Regularization of the divergent integrals. I. General consideration. Electronic Journal of Boundary Elements, 4(2), pp. 49-57, 2006.

[12] Zozulya V.V. Regularization of the divergent integrals. II. Application in Fracture Mechanics. Electronic Journal of Boundary Elements, 4(2), pp. 58-56, 2006.

[13] Zozulya V.V. Regularization of the hypersingular integrals in 3-D problems of fracture mechanics. In: Boundary Elements and Other Mesh Reduction Methods $X X X$. (Eds. P.Skerget and C.A. Brebbia), WIT Press, Southampton, Boston, 2008, 219-228. 
[14] Zozulya V.V. and Gonzalez-Chi P.I. Weakly singular, singular and hypersingular integrals in elasticity and fracture mechanics, Journal of the Chinese Institute of Engineers, 22(6), pp. 763-775, 1999.

[15] Zozulya V.V. and Lukin A.N. Solution of three-dimensional problems of fracture mechanics by the method of integral boundary equations. International Applied Mechanics, 34(6), pp. 544-551, 1998.

[16] Zozulya V.V. and Men'shikov V.A. Solution of tree dimensional problems of the dynamic theory of elasticity for bodies with cracks using hypersingular integrals, International Applied Mechanics, 36(1), pp. 74-81, 2000.

\section{$\underline{\text { Appendix A }}$}

\section{Regularization of the singular integrals related to the kernels $K_{i j}(\mathbf{x}, \mathbf{y})$}

$$
\text { Integral of the type } J_{2}^{1,0}=\int_{-1}^{1} \frac{x_{1}(\xi) \varphi_{q}(\xi)}{r\left(\xi, \mathbf{y}^{q}\right)^{2}} J(\xi) d \xi
$$

Node $q=0$ and $y_{1}^{0}=x_{1}^{0}$

$$
\begin{gathered}
L_{2}^{1,0}=\lim _{\xi \rightarrow-1} \frac{x_{1}(\xi) \varphi_{0}(\xi) J(\xi)(\xi+1)}{r\left(\xi, \mathbf{y}^{q}\right)^{2}}=-\frac{2 l_{1}^{0,2} S^{0,1}}{\left(l_{1}^{0,2}\right)^{2}+\left(l_{2}^{0,2}\right)^{2}}, \\
J_{2}^{1,0}=\int_{-1}^{1} \frac{x_{1}(\xi) \varphi_{0}(\xi)(\xi+1)-L_{2}^{1,0} r\left(\xi, \mathbf{y}^{q}\right)^{2}}{r\left(\xi, \mathbf{y}^{q}\right)^{2}(\xi+1)} J(\xi) d \xi+L_{2}^{1,0} \int_{-1}^{1} \frac{1}{\xi+1} d \xi .
\end{gathered}
$$

Node $q=1$ and $y_{1}^{1}=x_{1}^{1}$

$$
\begin{gathered}
L_{2}^{1,0}=\lim _{\xi \rightarrow 0} \frac{x_{1}(\xi) \varphi_{1}(\xi) J(\xi) \xi}{r\left(\xi, \mathbf{y}^{q}\right)^{2}}=\frac{\left(x_{1}^{2}-x_{1}^{0}\right)}{S^{0,2}}, \\
J_{2}^{1,0}=\int_{-1}^{1} \frac{x_{1}(\xi) \varphi_{1}(\xi) \xi-L_{2}^{1,0} r\left(\xi, \mathbf{y}^{q}\right)^{2}}{r\left(\xi, \mathbf{y}^{q}\right)^{2} \xi} J(\xi) d \xi+L_{2}^{1,0} \int_{-1}^{1} \frac{1}{\xi} d \xi .
\end{gathered}
$$

Node $q=2$ and $y_{1}^{2}=x_{1}^{2}$

$$
\begin{gathered}
L_{2}^{1,0}=\lim _{\xi \rightarrow 1} \frac{x_{1}(\xi) \varphi_{2}(\xi) J(\xi)(\xi-1)}{r\left(\xi, \mathbf{y}^{q}\right)^{2}}=-\frac{2 l_{1}^{2,0} S^{2,1}}{\left(l_{1}^{0,2}\right)^{2}+\left(l_{2}^{0,2}\right)^{2}}, \\
J_{2}^{1,0}=\int_{-1}^{1} \frac{x_{1}(\xi) \varphi_{2}(\xi)(\xi-1)-L_{2}^{1,0} r\left(\xi, \mathbf{y}^{q}\right)^{2}}{r\left(\xi, \mathbf{y}^{q}\right)^{2}(\xi-1)} J(\xi) d \xi+L_{2}^{1,0} \int_{-1}^{1} \frac{1}{\xi-1} d \xi .
\end{gathered}
$$

$$
\text { Integral of the type } J_{2}^{0,1}=\int_{-1}^{1} \frac{x_{2}(\xi) \varphi_{q}(\xi)}{r\left(\xi, \mathbf{y}^{q}\right)^{2}} J(\xi) d \xi
$$

Node $q=0$ and $y_{1}^{0}=x_{1}^{0}$

$$
L_{2}^{0,1}=\lim _{\xi \rightarrow-1} \frac{x_{2}(\xi) \varphi_{0}(\xi) J(\xi)(\xi+1)}{r\left(\xi, \mathbf{y}^{q}\right)^{2}}=-\frac{2 l_{2}^{0,2} S^{0,1}}{\left(l_{1}^{0,2}\right)^{2}+\left(l_{2}^{0,2}\right)^{2}},
$$




$$
J_{2}^{0,1}=\int_{-1}^{1} \frac{x_{2}(\xi) \varphi_{0}(\xi)(\xi+1)-L_{2}^{0,1} r\left(\xi, \mathbf{y}^{q}\right)^{2}}{r\left(\xi, \mathbf{y}^{q}\right)^{2}(\xi+1)} J(\xi) d \xi+L_{2}^{0,1} \int_{-1}^{1} \frac{1}{\xi+1} d \xi .
$$

Node $q=1$ and $y_{1}^{1}=x_{1}^{1}$

$$
\begin{gathered}
L_{2}^{0,1}=\lim _{\xi \rightarrow 0} \frac{x_{2}(\xi) \varphi_{1}(\xi) J(\xi) \xi}{r\left(\xi, \mathbf{y}^{q}\right)^{2}}=\frac{\left(x_{2}^{2}-x_{2}^{0}\right)}{S^{0,2}}, \\
J_{2}^{0,1}=\int_{-1}^{1} \frac{x_{2}(\xi) \varphi_{1}(\xi) \xi-L_{2}^{0,1} r\left(\xi, \mathbf{y}^{q}\right)^{2}}{r\left(\xi, \mathbf{y}^{q}\right)^{2} \xi} J(\xi) d \xi+L_{2}^{0,1} \int_{-1}^{1} \frac{1}{\xi} d \xi .
\end{gathered}
$$

Node $q=2$ and $y_{1}^{2}=x_{1}^{2}$

$$
\begin{gathered}
L_{2}^{0,1}=\lim _{\xi \rightarrow 1} \frac{\left.x_{2}(\xi) \varphi_{2}(\xi) J(\xi)\right)(\xi-1)}{r\left(\xi, \mathbf{y}^{q}\right)^{2}}=-\frac{2 l_{2}^{2,0} S^{2,1}}{\left(l_{1}^{0,2}\right)^{2}+\left(l_{2}^{0,2}\right)^{2}}, \\
J_{2}^{0,1}=\int_{-1}^{1} \frac{x_{2}(\xi) \varphi_{0}(\xi)(\xi-1)-L_{2}^{0,1} r\left(\xi, \mathbf{y}^{q}\right)^{2}}{r\left(\xi, \mathbf{y}^{q}\right)^{2}(\xi-1)} J(\xi) d \xi+L_{2}^{0,1} \int_{-1}^{1} \frac{1}{\xi-1} d \xi .
\end{gathered}
$$

Integral of the type $J_{4}^{3,0}=\int_{-1}^{1} \frac{x_{1}(\xi)^{3} \varphi_{q}(\xi)}{r\left(\xi, \mathbf{y}^{q}\right)^{4}} J(\xi) d \xi$

Node $q=0$ and $y_{1}^{0}=x_{1}^{0}$

$$
\begin{gathered}
L_{4}^{3,0}=\lim _{\xi \rightarrow-1} \frac{x_{1}(\xi)^{3} \varphi_{0}(\xi) J(\xi)(\xi+1)}{r\left(\xi, \mathbf{y}^{q}\right)^{4}}=-\frac{2\left(l_{1}^{0,2}\right)^{3} S^{0,1}}{\left(\left(l_{1}^{0,2}\right)^{2}+\left(l_{2}^{0,2}\right)^{2}\right)^{2}}, \\
J_{4}^{3,0}=\int_{-1}^{1} \frac{x_{1}(\xi)^{3} \varphi_{0}(\xi)(\xi+1)-L_{4}^{3,0} r\left(\xi, \mathbf{y}^{q}\right)^{4}}{r\left(\xi, \mathbf{y}^{q}\right)^{4}(\xi+1)} J(\xi) d \xi+L_{4}^{3,0} \int_{-1}^{1} \frac{1}{\xi+1} d \xi .
\end{gathered}
$$

Node $q=1$ and $y_{1}^{1}=x_{1}^{1}$

$$
\begin{gathered}
L_{4}^{3,0}=\lim _{\xi \rightarrow 0} \frac{x_{1}(\xi)^{3} \varphi_{1}(\xi) J(\xi) \xi}{r\left(\xi, \mathbf{y}^{q}\right)^{4}}=\left(\frac{\left(x_{1}^{2}-x_{1}^{0}\right)}{S^{0,2}}\right)^{3}, \\
J_{4}^{3,0}=I_{4}^{3,0}+L_{4}^{3,0} \int_{-1}^{1} \frac{1}{\xi} d \xi, I_{4}^{3,0}=\int_{-1}^{1} \frac{x_{1}(\xi)^{3} \varphi_{1}(\xi) \xi-L_{4}^{3,0} r\left(\xi, \mathbf{y}^{q}\right)^{4}}{r\left(\xi, \mathbf{y}^{q}\right)^{4} \xi} J(\xi) d \xi .
\end{gathered}
$$

Node $q=2$ and $y_{1}^{2}=x_{1}^{2}$

$$
\begin{gathered}
L_{4}^{3,0}=\lim _{\xi \rightarrow 1} \frac{x_{1}(\xi)^{3} \varphi_{2}(\xi) J(\xi)(\xi-1)}{r\left(\xi, \mathbf{y}^{q}\right)^{4}}=\frac{2\left(l_{1}^{2,0}\right)^{3} S^{2,1}}{\left(\left(l_{1}^{0,2}\right)^{2}+\left(l_{2}^{0,2}\right)^{2}\right)^{2}}, \\
J_{4}^{3,0}=\int_{-1}^{1} \frac{x_{1}(\xi)^{3} \varphi_{1}(\xi) \xi-L_{4}^{3,0} r\left(\xi, \mathbf{y}^{q}\right)^{4}}{r\left(\xi, \mathbf{y}^{q}\right)^{4} \xi} J(\xi) d \xi+L_{4}^{3,0} \int_{-1}^{1} \frac{1}{\xi-1} d \xi .
\end{gathered}
$$




$$
\text { Integral of the type } J_{4}^{0,3}=\int_{-1}^{1} \frac{x_{2}(\xi)^{3} \varphi_{q}(\xi)}{r\left(\xi, \mathbf{y}^{q}\right)^{4}} J(\xi) d \xi
$$

Node $q=0$ and $y_{1}^{0}=x_{1}^{0}$

$$
\begin{gathered}
L_{4}^{0,3}=\lim _{\xi \rightarrow-1} \frac{x_{2}(\xi)^{3} \varphi_{0}(\xi) J(\xi)(\xi+1)}{r\left(\xi, \mathbf{y}^{q}\right)^{4}}=-\frac{2\left(l_{2}^{0,2}\right)^{3} S^{0,1}}{\left(\left(l_{1}^{0,2}\right)^{2}+\left(l_{2}^{0,2}\right)^{2}\right)^{2}}, \\
J_{4}^{0,3}=\int_{-1}^{1} \frac{x_{2}(\xi)^{3} \varphi_{0}(\xi)(\xi+1)-L_{4}^{0,3} r\left(\xi, \mathbf{y}^{q}\right)^{4}}{r\left(\xi, \mathbf{y}^{q}\right)^{4}(\xi+1)} J(\xi) d \xi+L_{4}^{0,3} \int_{-1}^{1} \frac{1}{\xi+1} d \xi .
\end{gathered}
$$

Node $q=1$ and $y_{1}^{1}=x_{1}^{1}$

$$
\begin{gathered}
L_{4}^{0,3}=\lim _{\xi \rightarrow 0} \frac{x_{2}(\xi)^{3} \varphi_{1}(\xi) J(\xi) \xi}{r\left(\xi, \mathbf{y}^{q}\right)^{4}}=\left(\frac{\left(x_{2}^{2}-x_{2}^{0}\right)}{S^{0,2}}\right)^{3}, \\
J_{4}^{0,3}=\int_{-1}^{1} \frac{x_{2}(\xi)^{3} \varphi_{1}(\xi) \xi-L_{4}^{0,3} r\left(\xi, \mathbf{y}^{q}\right)^{4}}{r\left(\xi, \mathbf{y}^{q}\right)^{4} \xi} J(\xi) d \xi+L_{4}^{0,3} \int_{-1}^{1} \frac{1}{\xi} d \xi .
\end{gathered}
$$

Node $q=2$ and $y_{1}^{2}=x_{1}^{2}$

$$
\begin{gathered}
L_{4}^{0,3}=\lim _{\xi \rightarrow 1} \frac{x_{2}(\xi)^{3} \varphi_{2}(\xi) J(\xi)(\xi-1)}{r\left(\xi, \mathbf{y}^{q}\right)^{4}}=\frac{2\left(l_{2}^{2,0}\right)^{3} S^{2,1}}{\left(\left(l_{1}^{0,2}\right)^{2}+\left(l_{2}^{0,2}\right)^{2}\right)^{2}}, \\
J_{4}^{0,3}=\int_{-1}^{1} \frac{x_{2}(\xi)^{3} \varphi_{2}(\xi)(\xi-1)-L_{4}^{2,1} r\left(\xi, \mathbf{y}^{q}\right)^{4}}{r\left(\xi, \mathbf{y}^{q}\right)^{4}(\xi-1)} J(\xi) d \xi+L_{4}^{0,3} \int_{-1}^{1} \frac{1}{\xi-1} d \xi .
\end{gathered}
$$

$$
\text { Integral of the type } J_{4}^{2,1}=\int_{-1}^{1} \frac{x_{1}(\xi)^{2} x_{2}(\xi) \varphi_{q}(\xi)}{r\left(\xi, \mathbf{y}^{q}\right)^{4}} J(\xi) d \xi
$$

Node $q=0$ and $y_{1}^{0}=x_{1}^{0}$

$$
\begin{gathered}
L_{4}^{2,1}=\lim _{\xi \rightarrow-1} \frac{x_{1}(\xi)^{2} x_{2}(\xi) \varphi_{0}(\xi) J(\xi)(\xi+1)}{r\left(\xi, \mathbf{y}^{q}\right)^{4}}=-\frac{2\left(l_{1}^{0,2}\right)^{2} l_{2}^{0,2} S^{0,1}}{\left(\left(l_{1}^{0,2}\right)^{2}+\left(l_{2}^{0,2}\right)^{2}\right)^{2}}, \\
J_{4}^{2,1}=I_{4}^{2,1}+L_{4}^{2,1} \int_{-1}^{1} \frac{1}{\xi+1} d \xi, I_{4}^{2,1}=\int_{-1}^{1} \frac{x_{1}(\xi)^{2} x_{2}(\xi) \varphi_{0}(\xi)(\xi+1)-L_{4}^{2,1} r\left(\xi, \mathbf{y}^{q}\right)^{4}}{r\left(\xi, \mathbf{y}^{q}\right)^{4}(\xi+1)} J(\xi) d \xi .
\end{gathered}
$$

Node $q=1$ and $y_{1}^{1}=x_{1}^{1}$

$$
\begin{gathered}
L_{4}^{2,1}=\lim _{\xi \rightarrow 0} \frac{x_{1}(\xi)^{2} x_{2}(\xi) \varphi_{1}(\xi) J(\xi) \xi}{r\left(\xi, \mathbf{y}^{q}\right)^{4}}=\frac{\left(x_{1}^{2}-x_{1}^{0}\right)^{2}\left(x_{2}^{2}-x_{2}^{0}\right)}{\left(S^{0,2}\right)^{3}} \\
J_{4}^{2,1}=\int_{-1}^{1} \frac{x_{1}(\xi)^{2} x_{2}(\xi) \varphi_{0}(\xi)(\xi+1)-L_{4}^{2,1} r\left(\xi, \mathbf{y}^{q}\right)^{4}}{r\left(\xi, \mathbf{y}^{q}\right)^{4}(\xi+1)} J(\xi) d \xi+L_{4}^{2,1} \int_{-1}^{1} \frac{1}{\xi} d \xi .
\end{gathered}
$$

Node $q=2$ and $y_{1}^{2}=x_{1}^{2}$ 


$$
\begin{gathered}
L_{4}^{2,1}=\lim _{\xi \rightarrow 1} \frac{x_{1}(\xi)^{2} x_{2}(\xi) \varphi_{2}(\xi) J(\xi)(\xi-1)}{r\left(\xi, \mathbf{y}^{q}\right)^{4}}=\frac{2\left(l_{1}^{2,0}\right)^{2} l_{2}^{2,0} S^{2,1}}{\left(\left(l_{1}^{0,2}\right)^{2}+\left(l_{2}^{0,2}\right)^{2}\right)^{2}} \\
J_{4}^{2,1}=\int_{-1}^{1} \frac{x_{1}(\xi)^{2} x_{2}(\xi) \varphi_{2}(\xi)(\xi-1)-L_{4}^{2,1} r\left(\xi, \mathbf{y}^{q}\right)^{4}}{r\left(\xi, \mathbf{y}^{q}\right)^{4}(\xi-1)} J(\xi) d \xi+L_{4}^{2,1} \int_{-1}^{1} \frac{1}{\xi-1} d \xi .
\end{gathered}
$$

$$
\text { Integral of the type } J_{4}^{1,2}=\int_{-1}^{1} \frac{x_{1}(\xi) x_{2}(\xi)^{2} \varphi_{q}(\xi)}{r\left(\xi, \mathbf{y}^{q}\right)^{4}} J(\xi) d \xi
$$

Node $q=0$ and $y_{1}^{0}=x_{1}^{0}$

$$
\begin{gathered}
L_{4}^{1,2}=\lim _{\xi \rightarrow-1} \frac{x_{1}(\xi) x_{2}(\xi)^{2} \varphi_{0}(\xi) J(\xi)(\xi+1)}{r\left(\xi, \mathbf{y}^{q}\right)^{4}}=-\frac{2\left(l_{2}^{0,2}\right)^{2} l_{1}^{0,2} S^{0,1}}{\left(\left(l_{1}^{0,2}\right)^{2}+\left(l_{2}^{0,2}\right)^{2}\right)^{2}} \\
J_{4}^{1,2}=\int_{-1}^{1} \frac{x_{1}(\xi) x_{2}(\xi)^{2} \varphi_{0}(\xi)(\xi+1)-L_{4}^{1,2} r\left(\xi, \mathbf{y}^{q}\right)^{4}}{r\left(\xi, \mathbf{y}^{q}\right)^{4}(\xi+1)} J(\xi) d \xi+L_{4}^{1,2} \int_{-1}^{1} \frac{1}{\xi+1} d \xi .
\end{gathered}
$$

Node $q=1$ and $y_{1}^{1}=x_{1}^{1}$

$$
\begin{gathered}
L_{4}^{1,2}=\lim _{\xi \rightarrow 0} \frac{x_{1}(\xi) x_{2}(\xi)^{2} \varphi_{1}(\xi) J(\xi) \xi}{r\left(\xi, \mathbf{y}^{q}\right)^{4}}=\frac{\left(x_{1}^{2}-x_{1}^{0}\right)\left(x_{2}^{2}-x_{2}^{0}\right)^{2}}{\left(S^{0,2}\right)^{3}}, \\
J_{4}^{1,2}=\int_{-1}^{1} \frac{x_{1}(\xi) x_{2}(\xi)^{2} \varphi_{1}(\xi) \xi-L_{4}^{1,2} r\left(\xi, \mathbf{y}^{q}\right)^{4}}{r\left(\xi, \mathbf{y}^{q}\right)^{4} \xi} J(\xi) d \xi+L_{4}^{1,2} \int_{-1}^{1} \frac{1}{\xi} d \xi .
\end{gathered}
$$

Node $q=2$ and $y_{1}^{2}=x_{1}^{2}$

$$
\begin{gathered}
L_{4}^{1,2}=\lim _{\xi \rightarrow 1} \frac{x_{1}(\xi) x_{2}(\xi)^{2} \varphi_{2}(\xi) J(\xi)(\xi-1)}{r\left(\xi, \mathbf{y}^{q}\right)^{4}}=\frac{2\left(l_{2}^{2,0}\right)^{2} l_{1}^{2,0} S^{0,1}}{\left(\left(l_{1}^{0,2}\right)^{2}+\left(l_{2}^{0,2}\right)^{2}\right)^{2}} \\
J_{4}^{1,2}=\int_{-1}^{1} \frac{x_{1}(\xi) x_{2}(\xi)^{2} \varphi_{2}(\xi)(\xi-1)-L_{4}^{1,2} r\left(\xi, \mathbf{y}^{q}\right)^{4}}{r\left(\xi, \mathbf{y}^{q}\right)^{4}(\xi-1)} J(\xi) d \xi+L_{4}^{1,2} \int_{-1}^{1} \frac{1}{\xi-1} d \xi .
\end{gathered}
$$

where $S^{\alpha, \beta}=\sqrt{\left(x_{1}^{\alpha}-x_{1}^{\beta}\right)^{2}+\left(x_{1}^{\alpha}-x_{1}^{\beta}\right)^{2}}, l_{i}^{\alpha, \beta}=3 x_{1}^{\alpha}-4 x_{1}^{1}+x_{1}^{\beta}$.

\section{$\underline{\text { Appendix B }}$}

Regularization of the singular integrals related to the kernels $W_{i j}(\mathbf{x}, \mathbf{y})$

$$
\text { Integral of the type } J_{2}^{1,0}\left(n_{1}\right)=\int_{-1}^{1} \frac{x_{1}(\xi) \varphi_{q}(\xi) n_{1}(\xi)}{r\left(\xi, \mathbf{y}^{q}\right)^{2}} J(\xi) d \xi
$$

Node $q=0$ and $y_{1}^{0}=x_{1}^{0}$

$$
L_{2}^{1,0}=\lim _{\xi \rightarrow-1} \frac{x_{1}(\xi) \varphi_{0}(\xi) n_{1}(\xi) J(\xi)(\xi-1)}{r\left(\xi, \mathbf{y}^{q}\right)^{2}}=\frac{2 l_{1}^{0,2}\left(x_{2}^{0}-x_{2}^{1}\right)}{S_{1}+3 x_{1}^{0} m_{1}^{0,2}+3 x_{2}^{0} m_{2}^{0,2}},
$$




$$
J_{2}^{1,0}\left(n_{1}\right)=\int_{-1}^{1} \frac{x_{1}(\xi) \varphi_{0}(\xi) n_{1}(\xi)(\xi+1)-L_{2}^{1,0} r\left(\xi, \mathbf{y}^{q}\right)^{2}}{r\left(\xi, \mathbf{y}^{q}\right)^{2}(\xi+1)} J(\xi) d \xi+L_{2}^{1,0} \int_{-1}^{1} \frac{1}{\xi+1} d \xi .
$$

Node $q=1$ and $y_{1}^{1}=x_{1}^{1}$

$$
\begin{gathered}
L_{2}^{1,0}=\lim _{\xi \rightarrow 0} \frac{x_{1}(\xi) \varphi_{0}(\xi) n_{1}(\xi) J(\xi) \xi}{r\left(\xi, \mathbf{y}^{q}\right)^{2}}=\frac{\left(x_{1}^{2}-x_{1}^{0}\right)\left(x_{2}^{2}-x_{2}^{0}\right)}{\left(S^{0,2}\right)^{2}}, \\
J_{2}^{1,0}\left(n_{1}\right)=\int_{-1}^{1} \frac{x_{1}(\xi) \varphi_{1}(\xi) n_{1}(\xi) \xi-L_{2}^{1,0} r\left(\xi, \mathbf{y}^{q}\right)^{2}}{r\left(\xi, \mathbf{y}^{q}\right)^{2} \xi} J(\xi) d \xi+L_{2}^{1,0} \int_{-1}^{1} \frac{1}{\xi} d \xi .
\end{gathered}
$$

Node $q=2$ and $y_{1}^{2}=x_{1}^{2}$

$$
\begin{gathered}
L_{2}^{1,0}=\lim _{\xi \rightarrow 1} \frac{x_{1}(\xi) \varphi_{2}(\xi) n_{1}(\xi) J(\xi)(\xi-1)}{r\left(\xi, \mathbf{y}^{q}\right)^{2}}=\frac{2 l_{1}^{2,0}\left(x_{2}^{2}-x_{2}^{1}\right)}{S_{1}+3 x_{1}^{0} m_{1}^{0,2}+3 x_{2}^{0} m_{2}^{0,2}}, \\
J_{2}^{1,0}\left(n_{1}\right)=\int_{-1}^{1} \frac{x_{1}(\xi) \varphi_{2}(\xi) n_{1}(\xi)(\xi-1)-L_{2}^{1,0} r\left(\xi, \mathbf{y}^{q}\right)^{2}}{r\left(\xi, \mathbf{y}^{q}\right)^{2}(\xi-1)} J(\xi) d \xi+L_{2}^{1,0} \int_{-1}^{1} \frac{1}{\xi-1} d \xi .
\end{gathered}
$$

$$
\text { Integral of the type } J_{2}^{1,0}\left(n_{2}\right)=\int_{-1}^{1} \frac{x_{1}(\xi) \varphi_{q}(\xi) n_{2}(\xi)}{r\left(\xi, \mathbf{y}^{q}\right)^{2}} J(\xi) d \xi
$$

Node $q=0$ and $y_{1}^{0}=x_{1}^{0}$

$$
\begin{aligned}
L_{2}^{1,0} & =\lim _{\xi \rightarrow-1} \frac{x_{1}(\xi) \varphi_{0}(\xi) n_{2}(\xi) J(\xi)(\xi+1)}{r\left(\xi, \mathbf{y}^{q}\right)^{2}}=\frac{2 l_{1}^{0,2}\left(x_{1}^{0}-x_{1}^{1}\right)}{S_{1}+3 x_{1}^{0} m_{1}^{0,2}+3 x_{2}^{0} m_{2}^{0,2}}, \\
J_{2}^{1,0}\left(n_{2}\right) & =\int_{-1}^{1} \frac{x_{1}(\xi) \varphi_{0}(\xi) n_{2}(\xi)(\xi+1)-L_{2}^{1,0} r\left(\xi, \mathbf{y}^{q}\right)^{2}}{r\left(\xi, \mathbf{y}^{q}\right)^{2}(\xi+1)} J(\xi) d \xi+L_{2}^{1,0} \int_{-1}^{1} \frac{1}{\xi+1} d \xi .
\end{aligned}
$$

Node $q=1$ and $y_{1}^{1}=x_{1}^{1}$

$$
\begin{gathered}
L_{2}^{1,0}=\lim _{\xi \rightarrow 0} \frac{x_{1}(\xi) \varphi_{1}(\xi) n_{2}(\xi) J(\xi) \xi}{r\left(\xi, \mathbf{y}^{q}\right)^{2}}=\frac{\left(x_{1}^{0}-x_{1}^{2}\right)^{2}}{\left(S^{0,2}\right)^{2}}, \\
J_{2}^{1,0}\left(n_{2}\right)=\int_{-1}^{1} \frac{x_{1}(\xi) \varphi_{1}(\xi) n_{2}(\xi) \xi-L_{2}^{1,0} r\left(\xi, \mathbf{y}^{q}\right)^{2}}{r\left(\xi, \mathbf{y}^{q}\right)^{2} \xi} J(\xi) d \xi+L_{2}^{1,0} \int_{-1}^{1} \frac{1}{\xi} d \xi .
\end{gathered}
$$

Node $q=2$ and $y_{1}^{2}=x_{1}^{2}$

$$
\begin{gathered}
L_{2}^{1,0}=\lim _{\xi \rightarrow 1} \frac{x_{1}(\xi) \varphi_{2}(\xi) n_{2}(\xi) J(\xi)(\xi-1)}{r\left(\xi, \mathbf{y}^{q}\right)^{2}}=-\frac{2 l_{1}^{2,0}\left(x_{1}^{2}-x_{1}^{1}\right)}{S_{1}+3 x_{1}^{0} m_{1}^{0,2}+3 x_{2}^{0} m_{2}^{0,2}}, \\
J_{2}^{1,0}=\int_{-1}^{1} \frac{x_{1}(\xi) \varphi_{2}(\xi) n_{2}(\xi)(\xi-1)-L_{2}^{1,0} r\left(\xi, \mathbf{y}^{q}\right)^{2}}{r\left(\xi, \mathbf{y}^{q}\right)^{2}(\xi-1)} J(\xi) d \xi+L_{2}^{1,0} \int_{-1}^{1} \frac{1}{\xi-1} d \xi .
\end{gathered}
$$




$$
\text { Integral of the type } \left.J_{2}^{0,1}\left(n_{1}\right)\right)=\int_{-1}^{1} \frac{x_{2}(\xi) \varphi_{q}(\xi) n_{1}(\xi)}{r\left(\xi, \mathbf{y}^{q}\right)^{2}} J(\xi) d \xi
$$

Node $q=0$ and $y_{1}^{0}=x_{1}^{0}$

$$
\begin{aligned}
L_{2}^{0,1} & =\lim _{\xi \rightarrow-1} \frac{x_{2}(\xi) \varphi_{0}(\xi) n_{1}(\xi) J(\xi)(\xi+1)}{r\left(\xi, \mathbf{y}^{q}\right)^{2}}=-\frac{2 l_{2}^{0,2}\left(x_{2}^{0}-x_{2}^{1}\right)}{S_{1}+3 x_{1}^{0} m_{1}^{0,2}+3 x_{2}^{0} m_{2}^{0,2}}, \\
J_{2}^{0,1}\left(n_{1}\right) & =\int_{-1}^{1} \frac{x_{2}(\xi) \varphi_{0}(\xi) n_{1}(\xi)(\xi+1)-L_{2}^{0,1} r\left(\xi, \mathbf{y}^{q}\right)^{2}}{r\left(\xi, \mathbf{y}^{q}\right)^{2}(\xi+1)} J(\xi) d \xi+L_{2}^{0,1} \int_{-1}^{1} \frac{1}{\xi+1} d \xi .
\end{aligned}
$$

Node $q=1$ and $y_{1}^{1}=x_{1}^{1}$

$$
\begin{gathered}
L_{2}^{0,1}=\lim _{\xi \rightarrow 0} \frac{x_{2}(\xi) \varphi_{1}(\xi) n_{1}(\xi) J(\xi) \xi}{r\left(\xi, \mathbf{y}^{q}\right)^{2}}=\frac{\left(x_{2}^{0}-x_{2}^{2}\right)^{2}}{\left(S^{0,2}\right)^{2}}, \\
J_{2}^{0,1}\left(n_{1}\right)=\int_{-1}^{1} \frac{x_{2}(\xi) \varphi_{1}(\xi) n_{1}(\xi) \xi-L_{2}^{0,1} r\left(\xi, \mathbf{y}^{q}\right)^{2}}{r\left(\xi, \mathbf{y}^{q}\right)^{2} \xi} J(\xi) d \xi+L_{2}^{0,1} \int_{-1}^{1} \frac{1}{\xi} d \xi .
\end{gathered}
$$

Node $q=2$ and $y_{1}^{2}=x_{1}^{2}$

$$
\begin{gathered}
L_{2}^{0,1}=\lim _{\xi \rightarrow 1} \frac{x_{2}(\xi) \varphi_{2}(\xi) n_{1}(\xi) J(\xi)(\xi-1)}{r\left(\xi, \mathbf{y}^{q}\right)^{2}}=\frac{2 l_{2}^{2,0}\left(x_{2}^{2}-x_{2}^{1}\right)}{S_{1}+3 x_{1}^{0} m_{1}^{0,2}+3 x_{2}^{0} m_{2}^{0,2}} \\
J_{2}^{0,1}\left(n_{1}\right)=\int_{-1}^{1} \frac{x_{2}(\xi) \varphi_{2}(\xi) n_{1}(\xi)(\xi-1)-L_{2}^{0,1} r\left(\xi, \mathbf{y}^{q}\right)^{2}}{r\left(\xi, \mathbf{y}^{q}\right)^{2}(\xi-1)} J(\xi) d \xi+L_{2}^{0,1} \int_{-1}^{1} \frac{1}{\xi-1} d \xi .
\end{gathered}
$$

$$
\text { Integral of the type } J_{2}^{0,1}\left(n_{2}\right)=\int_{-1}^{1} \frac{x_{2}(\xi) \varphi_{q}(\xi) n_{2}(\xi)}{r\left(\xi, \mathbf{y}^{q}\right)^{2}} J(\xi) d \xi
$$

Node $q=0$ and $y_{1}^{0}=x_{1}^{0}$

$$
\begin{aligned}
L_{2}^{0,1} & =\lim _{\xi \rightarrow-1} \frac{x_{2}(\xi) \varphi_{0}(\xi) n_{2}(\xi) J(\xi)(\xi+1)}{r\left(\xi, \mathbf{y}^{q}\right)^{2}}=-\frac{2 l_{2}^{0,2}\left(x_{1}^{2}-x_{1}^{1}\right)}{S_{1}+3 x_{1}^{0} m_{1}^{0,2}+3 x_{2}^{0} m_{2}^{0,2}}, \\
J_{2}^{0,1}\left(n_{2}\right) & =\int_{-1}^{1} \frac{x_{2}(\xi) \varphi_{0}(\xi) n_{2}(\xi)(\xi+1)-L_{2}^{0,1} r\left(\xi, \mathbf{y}^{q}\right)^{2}}{r\left(\xi, \mathbf{y}^{q}\right)^{2}(\xi+1)} J(\xi) d \xi+L_{2}^{0,1} \int_{-1}^{1} \frac{1}{\xi+1} d \xi .
\end{aligned}
$$

Node $q=1$ and $y_{1}^{1}=x_{1}^{1}$

$$
\begin{aligned}
L_{2}^{0,1} & =\lim _{\xi \rightarrow 0} \frac{x_{2}(\xi) \varphi_{1}(\xi) n_{2}(\xi) J(\xi) \xi}{r\left(\xi, \mathbf{y}^{q}\right)^{2}}=-\frac{\left(x_{1}^{2}-x_{1}^{0}\right)\left(x_{2}^{2}-x_{2}^{0}\right)}{\left(S^{0,2}\right)^{2}}, \\
J_{2}^{0,1}\left(n_{2}\right) & =\int_{-1}^{1} \frac{x_{2}(\xi) \varphi_{1}(\xi) n_{2}(\xi) \xi-L_{2}^{0,1} r\left(\xi, \mathbf{y}^{q}\right)^{2}}{r\left(\xi, \mathbf{y}^{q}\right)^{2} \xi} J(\xi) d \xi+L_{2}^{1,0} \int_{-1}^{1} \frac{1}{\xi} d \xi .
\end{aligned}
$$

Node $q=2$ and $y_{1}^{2}=x_{1}^{2}$

$$
L_{2}^{0,1}=\lim _{\xi \rightarrow 1} \frac{x_{2}(\xi) \varphi_{1}(\xi) n_{2}(\xi) J(\xi)(\xi-1)}{r\left(\xi, \mathbf{y}^{q}\right)^{2}}=-\frac{2 l_{2}^{2,0}\left(x_{1}^{2}-x_{1}^{1}\right)}{S_{1}+3 x_{1}^{0} m_{1}^{0,2}+3 x_{2}^{0} m_{2}^{0,2}},
$$




$$
J_{2}^{0,1}\left(n_{2}\right)=\int_{-1}^{1} \frac{x_{2}(\xi) \varphi_{1}(\xi) n_{2}(\xi)(\xi-1)-L_{2}^{0,1} r\left(\xi, \mathbf{y}^{q}\right)^{2}}{r\left(\xi, \mathbf{y}^{q}\right)^{2}(\xi-1)} J(\xi) d \xi+L_{2}^{1,0} \int_{-1}^{1} \frac{1}{\xi-1} d \xi
$$

$$
\text { Integral of the type } J_{4}^{3,0}\left(n_{1}\right)=\int_{-1}^{1} \frac{x_{1}(\xi)^{3} \varphi_{q}(\xi) n_{1}(\xi)}{r\left(\xi, \mathbf{y}^{q}\right)^{4}} J(\xi) d \xi
$$

Node $q=0$ and $y_{1}^{0}=x_{1}^{0}$

$$
\begin{aligned}
L_{4}^{3,0} & =\lim _{\xi \rightarrow-1} \frac{x_{1}(\xi)^{3} \varphi_{0}(\xi) n_{1}(\xi) J(\xi)(\xi+1)}{r\left(\xi, \mathbf{y}^{q}\right)^{4}}=-\frac{2\left(l_{1}^{0,2}\right)^{3}\left(x_{2}^{2}-x_{2}^{1}\right)}{S_{1}+3 x_{1}^{0} m_{1}^{0,2}+3 x_{2}^{0} m_{2}^{0,2}}, \\
J_{4}^{3,0}\left(n_{1}\right) & =\int_{-1}^{1} \frac{x_{1}(\xi)^{3} \varphi_{0}(\xi) n_{1}(\xi)(\xi+1)-L_{4}^{3,0} r\left(\xi, \mathbf{y}^{q}\right)^{4}}{r\left(\xi, \mathbf{y}^{q}\right)^{4}(\xi+1)} J(\xi) d \xi+L_{4}^{3,0} \int_{-1}^{1} \frac{1}{\xi+1} d \xi .
\end{aligned}
$$

Node $q=1$ and $y_{1}^{1}=x_{1}^{1}$

$$
\begin{aligned}
L_{4}^{3,0} & =\lim _{\xi \rightarrow 0} \frac{x_{1}(\xi)^{3} \varphi_{1}(\xi) n_{1}(\xi) J(\xi) \xi}{r\left(\xi, \mathbf{y}^{q}\right)^{4}}=\frac{\left(x_{1}^{2}-x_{1}^{0}\right)^{3}\left(x_{2}^{2}-x_{2}^{0}\right)}{\left(S^{0,2}\right)^{2}}, \\
J_{4}^{3,0}\left(n_{1}\right) & =\int_{-1}^{1} \frac{x_{1}(\xi)^{3} \varphi_{1}(\xi) n_{1}(\xi) \xi-L_{4}^{3,0} r\left(\xi, \mathbf{y}^{q}\right)^{4}}{r\left(\xi, \mathbf{y}^{q}\right)^{4} \xi} J(\xi) d \xi+L_{4}^{3,0} \int_{-1}^{1} \frac{1}{\xi} d \xi .
\end{aligned}
$$

Node $q=2$ and $y_{1}^{2}=x_{1}^{2}$

$$
\begin{gathered}
L_{4}^{3,0}=\lim _{\xi \rightarrow 1} \frac{x_{1}(\xi)^{3} \varphi_{2}(\xi) n_{1}(\xi) J(\xi)(\xi-1)}{r\left(\xi, \mathbf{y}^{q}\right)^{4}}=\frac{2\left(l_{1}^{2,0}\right)^{3}\left(x_{2}^{2}-x_{2}^{1}\right)}{S_{1}+3 x_{1}^{0} m_{1}^{0,2}+3 x_{2}^{0} m_{2}^{0,2}}, \\
J_{4}^{3,0}\left(n_{1}\right)=\int_{-1}^{1} \frac{x_{1}(\xi)^{3} \varphi_{2}(\xi) n_{1}(\xi)(\xi-1)-L_{4}^{3,0} r\left(\xi, \mathbf{y}^{q}\right)^{4}}{r\left(\xi, \mathbf{y}^{q}\right)^{4}(\xi-1)} J(\xi) d \xi+L_{4}^{3,0} \int_{-1}^{1} \frac{1}{\xi-1} d \xi .
\end{gathered}
$$

Integral of the type $J_{4}^{0,3}\left(n_{2}\right)=\int_{-1}^{1} \frac{x_{2}(\xi)^{3} \varphi_{q}(\xi) n_{2}(\xi)}{r\left(\xi, \mathbf{y}^{q}\right)^{4}} J(\xi) d \xi$

Node $q=0$ and $y_{1}^{0}=x_{1}^{0}$

$$
\begin{gathered}
L_{4}^{0,3}=\lim _{\xi \rightarrow-1} \frac{x_{2}(\xi)^{3} \varphi_{0}(\xi) n_{2}(\xi) J(\xi)(\xi+1)}{r\left(\xi, \mathbf{y}^{q}\right)^{4}}=\frac{2\left(l_{2}^{0,2}\right)^{3}\left(x_{1}^{2}-x_{1}^{1}\right)}{S_{1}+3 x_{1}^{0} m_{1}^{0,2}+3 x_{2}^{0} m_{2}^{0,2}}, \\
J_{4}^{0,3}\left(n_{2}\right)=\int_{-1}^{1} \frac{x_{2}(\xi)^{3} \varphi_{0}(\xi) n_{2}(\xi)(\xi+1)-L_{4}^{0,3} r\left(\xi, \mathbf{y}^{q}\right)^{4}}{r\left(\xi, \mathbf{y}^{q}\right)^{4}(\xi+1)} J(\xi) d \xi+L_{4}^{0,3} \int_{-1}^{1} \frac{1}{\xi+1} d \xi .
\end{gathered}
$$

Node $q=1$ and $y_{1}^{1}=x_{1}^{1}$

$$
\begin{aligned}
L_{4}^{0,3} & =\lim _{\xi \rightarrow 0} \frac{x_{2}(\xi)^{3} \varphi_{1}(\xi) n_{2}(\xi) J(\xi) \xi}{r\left(\xi, \mathbf{y}^{q}\right)^{4}}=-\frac{\left(x_{1}^{0}-x_{1}^{2}\right)\left(x_{2}^{0}-x_{2}^{2}\right)^{3}}{\left(S^{0,2}\right)^{2}}, \\
J_{4}^{0,3}\left(n_{2}\right) & =\int_{-1}^{1} \frac{x_{2}(\xi)^{3} \varphi_{1}(\xi) n_{2}(\xi) \xi-L_{4}^{0,3} r\left(\xi, \mathbf{y}^{q}\right)^{4}}{r\left(\xi, \mathbf{y}^{q}\right)^{4} \xi} J(\xi) d \xi+L_{4}^{0,3} \int_{-1}^{1} \frac{1}{\xi} d \xi .
\end{aligned}
$$


Node $q=2$ and $y_{1}^{2}=x_{1}^{2}$

$$
\begin{aligned}
L_{4}^{0,3} & =\lim _{\xi \rightarrow 1} \frac{x_{2}(\xi)^{3} \varphi_{2}(\xi) n_{2}(\xi) J(\xi)(\xi-1)}{r\left(\xi, \mathbf{y}^{q}\right)^{4}}=-\frac{2\left(l_{1}^{2,0}\right)^{3}\left(x_{1}^{2}-x_{1}^{1}\right)}{S_{1}+3 x_{1}^{0} m_{1}^{0,2}+3 x_{2}^{0} m_{2}^{0,2}}, \\
J_{4}^{0,3}\left(n_{2}\right) & =\int_{-1}^{1} \frac{x_{2}(\xi)^{3} \varphi_{2}(\xi) n_{2}(\xi)(\xi-1)-L_{4}^{0,3} r\left(\xi, \mathbf{y}^{q}\right)^{4}}{r\left(\xi, \mathbf{y}^{q}\right)^{4}(\xi-1)} J(\xi) d \xi+L_{4}^{0,3} \int_{-1}^{1} \frac{1}{\xi-1} d \xi .
\end{aligned}
$$

Integral of the type $J_{4}^{2,1}\left(n_{1}\right)=\int_{-1}^{1} \frac{x_{1}(\xi)^{2} x_{2}(\xi) \varphi_{q}(\xi) n_{1}(\xi)}{r\left(\xi, \mathbf{y}^{q}\right)^{4}} J(\xi) d \xi$

Node $q=0$ and $y_{1}^{0}=x_{1}^{0}$

$$
\begin{gathered}
L_{4}^{2,1}=\lim _{\xi \rightarrow-1} \frac{x_{1}(\xi)^{2} x_{2}(\xi) \varphi_{0}(\xi) n_{1}(\xi) J(\xi)(\xi+1)}{r\left(\xi, \mathbf{y}^{q}\right)^{4}}=\frac{2\left(l_{1}^{0,2}\right)^{2} l_{2}^{0,2}\left(x_{2}^{2}-x_{2}^{1}\right)}{S_{1}+3 x_{1}^{0} m_{1}^{0,2}+3 x_{2}^{0} m_{2}^{0,2}}, \\
J_{4}^{2,1}=\int_{-1}^{1} \frac{x_{1}(\xi)^{2} x_{2}(\xi) \varphi_{0}(\xi) n_{1}(\xi)(\xi+1)-L_{4}^{2,1} r\left(\xi, \mathbf{y}^{q}\right)^{4}}{r\left(\xi, \mathbf{y}^{q}\right)^{4}(\xi+1)} J(\xi) d \xi+L_{4}^{2,1} \int_{-1}^{1} \frac{1}{\xi+1} d \xi .
\end{gathered}
$$

Node $q=1$ and $y_{1}^{1}=x_{1}^{1}$

$$
\begin{aligned}
L_{4}^{2,1} & =\lim _{\xi \rightarrow 0} \frac{x_{1}(\xi)^{2} x_{2}(\xi) \varphi_{1}(\xi) n_{1}(\xi) J(\xi) \xi}{r\left(\xi, \mathbf{y}^{q}\right)^{4}}=\frac{\left(x_{1}^{0}-x_{1}^{2}\right)^{2}\left(x_{2}^{0}-x_{2}^{2}\right)^{2}}{\left(S^{0,2}\right)^{2}} \\
J_{4}^{2,1}\left(n_{1}\right) & =\int_{-1}^{1} \frac{x_{1}(\xi)^{2} x_{2}(\xi) n_{1}(\xi) \varphi_{1}(\xi) \xi-L_{4}^{2,1} r\left(\xi, \mathbf{y}^{q}\right)^{4}}{r\left(\xi, \mathbf{y}^{q}\right)^{4} \xi} J(\xi) d \xi+L_{4}^{2,1} \int_{-1}^{1} \frac{1}{\xi} d \xi .
\end{aligned}
$$

Node $q=2$ and $y_{1}^{2}=x_{1}^{2}$

$$
\begin{gathered}
L_{4}^{2,1}=\lim _{\xi \rightarrow 1} \frac{x_{1}(\xi)^{2} x_{2}(\xi) \varphi_{2}(\xi) n_{1}(\xi) J(\xi)(\xi-1)}{r\left(\xi, \mathbf{y}^{q}\right)^{4}}=\frac{2\left(l_{1}^{2,0}\right)^{2} l_{2}^{2,0}\left(x_{2}^{2}-x_{2}^{1}\right)}{S_{1}+3 x_{1}^{0} m_{1}^{0,2}+3 x_{2}^{0} m_{2}^{0,2}}, \\
J_{4}^{2,1}\left(n_{1}\right)=\int_{-1}^{1} \frac{x_{1}(\xi)^{2} x_{2}(\xi) \varphi_{2}(\xi) n_{1}(\xi)(\xi-1)-L_{4}^{2,1} r\left(\xi, \mathbf{y}^{q}\right)^{4}}{r\left(\xi, \mathbf{y}^{q}\right)^{4}(\xi-1)} J(\xi) d \xi+L_{4}^{2,1} \int_{-1}^{1} \frac{1}{\xi-1} d \xi .
\end{gathered}
$$

Integral of the type $J_{4}^{2,1}\left(n_{2}\right)=\int_{-1}^{1} \frac{x_{1}(\xi)^{2} x_{2}(\xi) \varphi_{q}(\xi) n_{2}(\xi)}{r\left(\xi, \mathbf{y}^{q}\right)^{4}} J(\xi) d \xi$

Node $q=0$ and $y_{1}^{0}=x_{1}^{0}$

$$
\begin{gathered}
L_{4}^{2,1}=\lim _{\xi \rightarrow-1} \frac{x_{1}(\xi)^{2} x_{2}(\xi) \varphi_{0}(\xi) n_{2}(\xi) J(\xi)(\xi+1)}{r\left(\xi, \mathbf{y}^{q}\right)^{4}}=-\frac{2\left(l_{1}^{0,2}\right)^{2} l_{2}^{0,2}\left(x_{1}^{2}-x_{1}^{1}\right)}{S_{1}+3 x_{1}^{0} m_{1}^{0,2}+3 x_{2}^{0} m_{2}^{0,2}}, \\
J_{4}^{2,1}=\int_{-1}^{1} \frac{x_{1}(\xi)^{2} x_{2}(\xi) \varphi_{0}(\xi) n_{2}(\xi)(\xi+1)-L_{4}^{2,1} r\left(\xi, \mathbf{y}^{q}\right)^{4}}{r\left(\xi, \mathbf{y}^{q}\right)^{4}(\xi+1)} J(\xi) d \xi+L_{4}^{2,1} \int_{-1}^{1} \frac{1}{\xi+1} d \xi .
\end{gathered}
$$

Node $q=1$ and $y_{1}^{1}=x_{1}^{1}$ 


$$
\begin{aligned}
L_{4}^{2,1} & =\lim _{\xi \rightarrow 0} \frac{x_{1}(\xi)^{2} x_{2}(\xi) \varphi_{1}(\xi) n_{2}(\xi) J(\xi) \xi}{r\left(\xi, \mathbf{y}^{q}\right)^{4}}=-\frac{\left(x_{1}^{0}-x_{1}^{2}\right)^{2}\left(x_{2}^{0}-x_{2}^{2}\right)^{2}}{\left(S^{0,2}\right)^{2}}, \\
J_{4}^{2,1}\left(n_{2}\right) & =\int_{-1}^{1} \frac{x_{1}(\xi)^{2} x_{2}(\xi) \varphi_{1}(\xi) n_{2}(\xi) \xi-L_{4}^{2,1} r\left(\xi, \mathbf{y}^{q}\right)^{4}}{r\left(\xi, \mathbf{y}^{q}\right)^{4} \xi} J(\xi) d \xi+L_{4}^{2,1} \int_{-1}^{1} \frac{1}{\xi} d \xi .
\end{aligned}
$$

Node $q=2$ and $y_{1}^{2}=x_{1}^{2}$

$$
\begin{aligned}
L_{4}^{2,1} & =\lim _{\xi \rightarrow 1} \frac{x_{1}(\xi)^{2} x_{2}(\xi) \varphi_{2}(\xi) n_{2}(\xi) J(\xi)(\xi-1)}{r\left(\xi, \mathbf{y}^{q}\right)^{4}}=-\frac{2\left(l_{1}^{2,0}\right)^{2} l_{2}^{2,0}\left(x_{1}^{2}-x_{1}^{1}\right)}{S_{1}+3 x_{1}^{0} m_{1}^{0,2}+3 x_{2}^{0} m_{2}^{0,2}}, \\
J_{4}^{2,1}\left(n_{2}\right) & =\int_{-1}^{1} \frac{x_{1}(\xi)^{2} x_{2}(\xi) \varphi_{2}(\xi) n_{2}(\xi)(\xi-1)-L_{4}^{2,1} r\left(\xi, \mathbf{y}^{q}\right)^{4}}{r\left(\xi, \mathbf{y}^{q}\right)^{4}(\xi-1)} J(\xi) d \xi+L_{4}^{2,1} \int_{-1}^{1} \frac{1}{\xi-1} d \xi .
\end{aligned}
$$

$$
\text { Integral of the type } J_{4}^{1,2}\left(n_{1}\right)=\int_{-1}^{1} \frac{x_{1}(\xi) x_{2}(\xi)^{2} \varphi_{q}(\xi) n_{1}(\xi)}{r\left(\xi, \mathbf{y}^{q}\right)^{4}} J(\xi) d \xi
$$

Node $q=0$ and $y_{1}^{0}=x_{1}^{0}$

$$
\begin{gathered}
L_{4}^{1,2}=\lim _{\xi \rightarrow-1} \frac{x_{1}(\xi) x_{2}(\xi)^{2} \varphi_{0}(\xi) n_{1}(\xi) J(\xi)(\xi+1)}{r\left(\xi, \mathbf{y}^{q}\right)^{4}}=\frac{2\left(l_{1}^{0,2}\right)^{2} l_{2}^{0,2}\left(x_{2}^{2}-x_{2}^{1}\right)}{S_{1}+3 x_{1}^{0} m_{1}^{0,2}+3 x_{2}^{0} m_{2}^{0,2}}, \\
J_{4}^{1,2}\left(n_{1}\right)=I_{4}^{1,2}=\int_{-1}^{1} \frac{x_{1}(\xi) x_{2}(\xi)^{2} \varphi_{0}(\xi) n_{1}(\xi)(\xi+1)-L_{4}^{1,2} r\left(\xi, \mathbf{y}^{q}\right)^{4}}{r\left(\xi, \mathbf{y}^{q}\right)^{4}(\xi+1)} J(\xi) d \xi+L_{4}^{1,2} \int_{-1}^{1} \frac{1}{\xi+1} d \xi .
\end{gathered}
$$

Node $q=1$ and $y_{1}^{1}=x_{1}^{1}$

$$
\begin{aligned}
L_{4}^{1,2} & =\lim _{\xi \rightarrow 0} \frac{x_{1}(\xi) x_{2}(\xi)^{2} \varphi_{1}(\xi) n_{1}(\xi) J(\xi) \xi}{r\left(\xi, \mathbf{y}^{q}\right)^{4}}=-\frac{\left(x_{1}^{0}-x_{1}^{2}\right)\left(x_{2}^{0}-x_{2}^{2}\right)^{2}}{\left(S^{0,2}\right)^{2}}, \\
J_{4}^{1,2}\left(n_{1}\right) & =\int_{-1}^{1} \frac{x_{1}(\xi) x_{2}(\xi)^{2} \varphi_{1}(\xi) n_{1}(\xi) \xi-L_{4}^{1,2} r\left(\xi, \mathbf{y}^{q}\right)^{4}}{r\left(\xi, \mathbf{y}^{q}\right)^{4} \xi} J(\xi) d \xi+L_{4}^{1,2} \int_{-1}^{1} \frac{1}{\xi} d \xi .
\end{aligned}
$$

Node $q=2$ and $y_{1}^{2}=x_{1}^{2}$

$$
\begin{aligned}
L_{4}^{1,2} & =\lim _{\xi \rightarrow 1} \frac{x_{1}(\xi) x_{2}(\xi)^{2} \varphi_{2}(\xi) n_{1}(\xi) J(\xi)(\xi-1)}{r\left(\xi, \mathbf{y}^{q}\right)^{4}}=\frac{2\left(l_{1}^{2,0}\right)^{2} l_{2}^{2,0}\left(x_{1}^{2}-x_{1}^{1}\right)}{S_{1}+3 x_{1}^{0} m_{1}^{0,2}+3 x_{2}^{0} m_{2}^{0,2}}, \\
J_{4}^{1,2}\left(n_{1}\right) & =\int_{-1}^{1} \frac{x_{1}(\xi) x_{2}(\xi)^{2} \varphi_{2}(\xi) n_{1}(\xi)(\xi-1)-L_{4}^{1,2} r\left(\xi, \mathbf{y}^{q}\right)^{4}}{r\left(\xi, \mathbf{y}^{q}\right)^{4}(\xi-1)} J(\xi) d \xi+L_{4}^{1,2} \int_{-1}^{1} \frac{1}{\xi-1} d \xi .
\end{aligned}
$$

Integral of the type $J_{4}^{1,2}\left(n_{2}\right)=\int_{-1}^{1} \frac{x_{1}(\xi) x_{2}(\xi)^{2} \varphi_{q}(\xi) n_{2}(\xi)}{r\left(\xi, \mathbf{y}^{q}\right)^{4}} J(\xi) d \xi$

Node $q=0$ and $y_{1}^{0}=x_{1}^{0}$ 


$$
\begin{gathered}
L_{4}^{1,2}=\lim _{\xi \rightarrow-1} \frac{x_{1}(\xi) x_{2}(\xi)^{2} \varphi_{0}(\xi) n_{2}(\xi) J(\xi)(\xi+1)}{r\left(\xi, \mathbf{y}^{q}\right)^{4}}=-\frac{2 l_{1}^{0,2}\left(l_{2}^{0,2}\right)^{2}\left(x_{1}^{2}-x_{1}^{1}\right)}{S_{1}+3 x_{1}^{0} m_{1}^{0,2}+3 x_{2}^{0} m_{2}^{0,2}}, \\
J_{4}^{1,2}=\int_{-1}^{1} \frac{x_{1}(\xi) x_{2}(\xi)^{2} \varphi_{0}(\xi) n_{2}(\xi)(\xi+1)-L_{4}^{1,2} r\left(\xi, \mathbf{y}^{q}\right)^{4}}{r\left(\xi, \mathbf{y}^{q}\right)^{4}(\xi+1)} J(\xi) d \xi+L_{4}^{1,2} \int_{-1}^{1} \frac{1}{\xi+1} d \xi .
\end{gathered}
$$

Node $q=1$ and $y_{1}^{1}=x_{1}^{1}$

$$
\begin{aligned}
L_{4}^{1,2} & =\lim _{\xi \rightarrow 0} \frac{x_{1}(\xi) x_{2}(\xi)^{2} \varphi_{1}(\xi) n_{2}(\xi) J(\xi) \xi}{r\left(\xi, \mathbf{y}^{q}\right)^{4}}=-\frac{\left(x_{1}^{0}-x_{1}^{2}\right)^{2}\left(x_{2}^{0}-x_{2}^{2}\right)}{\left(S^{0,2}\right)^{2}}, \\
J_{4}^{1,2}\left(n_{2}\right) & =\int_{-1}^{1} \frac{x_{1}(\xi) x_{2}(\xi)^{2} \varphi_{1}(\xi) n_{2}(\xi) \xi-L_{4}^{1,2} r\left(\xi, \mathbf{y}^{q}\right)^{4}}{r\left(\xi, \mathbf{y}^{q}\right)^{4} \xi} J(\xi) d \xi+L_{4}^{1,2} \int_{-1}^{1} \frac{1}{\xi} d \xi .
\end{aligned}
$$

Node $q=2$ and $y_{1}^{2}=x_{1}^{2}$

$$
\begin{gathered}
L_{4}^{1,2} \lim _{\xi \rightarrow 1} \frac{x_{1}(\xi) x_{2}(\xi)^{2} \varphi_{2}(\xi) n_{2}(\xi) J(\xi)(\xi-1)}{r\left(\xi, \mathbf{y}^{q}\right)^{4}}=-\frac{2 l_{1}^{2,0}\left(l_{2}^{2,0}\right)^{2}\left(x_{2}^{2}-x_{2}^{1}\right)}{S_{1}+3 x_{1}^{0} m_{1}^{0,2}+3 x_{2}^{0} m_{2}^{0,2}}, \\
J_{4}^{1,2}\left(n_{2}\right)=\int_{-1}^{1} \frac{x_{1}(\xi) x_{2}(\xi)^{2} \varphi_{2}(\xi) n_{2}(\xi)(\xi-1)-L_{4}^{1,2} r\left(\xi, \mathbf{y}^{q}\right)^{4}}{r\left(\xi, \mathbf{y}^{q}\right)^{4}(\xi-1)} J(\xi) d \xi+L_{4}^{1,2} \int_{-1}^{1} \frac{1}{\xi-1} d \xi .
\end{gathered}
$$

Here $S_{1}=\left(4 x_{1}^{1}-x_{1}^{2}\right)^{2}+\left(4 x_{2}^{1}-x_{2}^{2}\right)^{2}, m_{i}^{\alpha, \beta}=3 x_{i}^{\alpha}-8 x_{i}^{1}+2 x_{i}^{\beta}$.

\section{$\underline{\text { Appendix C }}$}

Regularization of the hypersingular integrals related to the kernels $F_{i j}(\mathbf{x}, \mathbf{y})$

$$
\text { Integral of the type } J_{2}\left(n_{1}\right)=\int_{-1}^{1} \frac{\varphi_{q}(\xi) n_{1}(\xi)}{r\left(\xi, \mathbf{y}^{q}\right)^{2}} J(\xi) d \xi
$$

Node $q=0$ and $y_{1}^{0}=x_{1}^{0}$

$$
\begin{gathered}
L_{2}=\lim _{\xi \rightarrow-1} \frac{\varphi_{0}(\xi) n_{1}(\xi) J(\xi)(\xi-1)^{2}}{r\left(\xi, \mathbf{y}^{q}\right)^{2}}=-\frac{4\left(x_{2}^{0}-x_{2}^{1}\right)}{S_{2}+x_{1}^{2} n_{1}^{0,2}+x_{2}^{2} n_{2}^{0,2}}, \\
J_{2}\left(n_{1}\right)=\int_{-1}^{1} \frac{\varphi_{0}(\xi) n_{1}(\xi)(\xi+1)^{2}-L_{2} r\left(\xi, \mathbf{y}^{q}\right)^{2}}{r\left(\xi, \mathbf{y}^{q}\right)^{2}(\xi+1)^{2}} J(\xi) d \xi+L_{2} \int_{-1}^{1} \frac{1}{(\xi+1)^{2}} d \xi .
\end{gathered}
$$

Node $q=1$ and $y_{1}^{1}=x_{1}^{1}$

$$
\begin{gathered}
L_{2}=\lim _{\xi \rightarrow 0} \frac{\varphi_{1}(\xi) n_{1}(\xi) J(\xi) \xi^{2}}{r\left(\xi, \mathbf{y}^{q}\right)^{2}}=-\frac{2\left(x_{1}^{0}-x_{1}^{2}\right)}{\left(S^{0,2}\right)^{2}}, \\
J_{2}\left(n_{1}\right)=\int_{-1}^{1} \frac{\varphi_{1}(\xi) n_{1}(\xi) \xi^{2}-L_{2} r\left(\xi, \mathbf{y}^{q}\right)^{2}}{r\left(\xi, \mathbf{y}^{q}\right)^{2} \xi^{2}} J(\xi) d \xi+L_{2} \int_{-1}^{1} \frac{1}{\xi^{2}} d \xi .
\end{gathered}
$$

Node $q=2$ and $y_{1}^{2}=x_{1}^{2}$ 


$$
\begin{gathered}
L_{2}=\lim _{\xi \rightarrow-1} \frac{\varphi_{2}(\xi) n_{1}(\xi) J(\xi)(\xi-1)^{2}}{r\left(\xi, \mathbf{y}^{q}\right)^{2}}=\frac{4\left(x_{2}^{2}-x_{2}^{1}\right)}{S_{2}+x_{1}^{0} n_{1}^{0,2}+x_{2}^{0} n_{2}^{0,2}}, \\
J_{2}\left(n_{1}\right)=\int_{-1}^{1} \frac{\varphi_{2}(\xi) n_{1}(\xi)(\xi+1)^{2}-L_{2} r\left(\xi, \mathbf{y}^{q}\right)^{2}}{r\left(\xi, \mathbf{y}^{q}\right)^{2}(\xi+1)^{2}} J(\xi) d \xi+L_{2} \int_{-1}^{1} \frac{1}{(\xi+1)^{2}} d \xi .
\end{gathered}
$$

$$
\text { Integral of the type } J_{2}\left(n_{2}\right)=\int_{-1}^{1} \frac{\varphi_{q}(\xi) n_{2}(\xi)}{r\left(\xi, \mathbf{y}^{q}\right)^{2}} J(\xi) d \xi
$$

Node $q=0$ and $y_{1}^{0}=x_{1}^{0}$

$$
\begin{gathered}
L_{2}=\lim _{\xi \rightarrow-1} \frac{\varphi_{0}(\xi) n_{2}(\xi) J(\xi)(\xi-1)^{2}}{r\left(\xi, \mathbf{y}^{q}\right)^{2}}=\frac{4\left(x_{1}^{0}-x_{1}^{1}\right)}{S_{2}+x_{1}^{2} n_{1}^{0,2}+x_{2}^{2} n_{2}^{0,2}}, \\
J_{2}\left(n_{1}\right)=\int_{-1}^{1} \frac{\varphi_{0}(\xi) n_{1}(\xi)(\xi+1)^{2}-L_{2} r\left(\xi, \mathbf{y}^{q}\right)^{2}}{r\left(\xi, \mathbf{y}^{q}\right)^{2}(\xi+1)^{2}} J(\xi) d \xi+L_{2} \int_{-1}^{1} \frac{1}{(\xi+1)^{2}} d \xi .
\end{gathered}
$$

Node $q=1$ and $y_{1}^{1}=x_{1}^{1}$

$$
\begin{gathered}
L_{2}=\lim _{\xi \rightarrow 0} \frac{\varphi_{1}(\xi) n_{1}(\xi) J(\xi) \xi^{2}}{r\left(\xi, \mathbf{y}^{q}\right)^{2}}=\frac{2\left(x_{2}^{0}-x_{2}^{2}\right)}{\left(S^{0,2}\right)^{2}}, \\
J_{2}\left(n_{1}\right)=\int_{-1}^{1} \frac{\varphi_{1}(\xi) n_{1}(\xi) \xi^{2}-L_{2} r\left(\xi, \mathbf{y}^{q}\right)^{2}}{r\left(\xi, \mathbf{y}^{q}\right)^{2} \xi^{2}} J(\xi) d \xi+L_{2} \int_{-1}^{1} \frac{1}{\xi^{2}} d \xi .
\end{gathered}
$$

Node $q=2$ and $y_{1}^{2}=x_{1}^{2}$

$$
\begin{gathered}
L_{2}=\lim _{\xi \rightarrow-1} \frac{\varphi_{2}(\xi) n_{1}(\xi) J(\xi)(\xi-1)^{2}}{r\left(\xi, \mathbf{y}^{q}\right)^{2}}=\frac{-4\left(x_{2}^{2}-x_{2}^{1}\right)}{S_{2}+x_{1}^{0} n_{1}^{0,2}+x_{2}^{0} n_{2}^{0,2}}, \\
J_{2}\left(n_{1}\right)=\int_{-1}^{1} \frac{\varphi_{2}(\xi) n_{1}(\xi)(\xi+1)^{2}-L_{2} r\left(\xi, \mathbf{y}^{q}\right)^{2}}{r\left(\xi, \mathbf{y}^{q}\right)^{2}(\xi+1)^{2}} J(\xi) d \xi+L_{2} \int_{-1}^{1} \frac{1}{(\xi+1)^{2}} d \xi .
\end{gathered}
$$

Integral of the type $J_{4}^{2,0}\left(n_{1}\right)=\int_{-1}^{1} \frac{\varphi_{q}(\xi) x_{1}(\xi)^{2} n_{1}(\xi)}{r\left(\xi, \mathbf{y}^{q}\right)^{4}} J(\xi) d \xi$

Node $q=0$ and $y_{1}^{0}=x_{1}^{0}$

$$
\begin{gathered}
L_{4}^{2,0}=\lim _{\xi \rightarrow-1} \frac{\varphi_{0}(\xi) x_{1}(\xi)^{2} n_{1}(\xi) J(\xi)(\xi-1)^{2}}{r\left(\xi, \mathbf{y}^{q}\right)^{4}}=-\frac{4\left(x_{2}^{0}-x_{2}^{1}\right)\left(l_{1}^{0,2}\right)^{2}}{\left(S_{2}+x_{1}^{2} n_{1}^{0,2}+x_{2}^{2} n_{2}^{0,2}\right)^{2}}, \\
J_{4}^{2,0}\left(n_{1}\right)=\int_{-1}^{1} \frac{\varphi_{0}(\xi) x_{1}(\xi)^{2} n_{1}(\xi)(\xi+1)^{2}-L_{4}^{2,0} r\left(\xi, \mathbf{y}^{q}\right)^{4}}{r\left(\xi, \mathbf{y}^{q}\right)^{4}(\xi+1)^{2}} J(\xi) d \xi+L_{4}^{2,0} \int_{-1}^{1} \frac{1}{(\xi+1)^{2}} d \xi .
\end{gathered}
$$

Node $q=1$ and $y_{1}^{1}=x_{1}^{1}$

$$
L_{4}^{2,0}=\lim _{\xi \rightarrow 0} \frac{\varphi_{1}(\xi) x_{1}(\xi)^{2} n_{1}(\xi) J(\xi) \xi^{2}}{r\left(\xi, \mathbf{y}^{q}\right)^{4}}=-\frac{2\left(x_{1}^{0}-x_{1}^{2}\right)^{2}\left(x_{2}^{0}-x_{2}^{2}\right)}{\left(S^{0,2}\right)^{2}},
$$




$$
J_{4}^{2,0}\left(n_{1}\right)=\int_{-1}^{1} \frac{\varphi_{1}(\xi) x_{1}(\xi)^{2} n_{1}(\xi) \xi^{2}-L_{4}^{2,0} r\left(\xi, \mathbf{y}^{q}\right)^{4}}{r\left(\xi, \mathbf{y}^{q}\right)^{4} \xi^{2}} J(\xi) d \xi+L_{4}^{2,0} \int_{-1}^{1} \frac{1}{\xi^{2}} d \xi .
$$

Node $q=2$ and $y_{1}^{2}=x_{1}^{2}$

$$
\begin{gathered}
L_{4}^{2,0}=\lim _{\xi \rightarrow-1} \frac{\varphi_{2}(\xi) x_{1}(\xi)^{2} n_{1}(\xi) J(\xi)(\xi-1)^{2}}{r\left(\xi, \mathbf{y}^{q}\right)^{4}}=\frac{4\left(x_{2}^{2}-x_{2}^{1}\right)\left(l_{1}^{0,2}\right)^{2}}{\left(S_{2}+x_{1}^{2} n_{1}^{0,2}+x_{2}^{2} n_{2}^{0,2}\right)^{2}}, \\
J_{4}^{2,0}\left(n_{1}\right)=\int_{-1}^{1} \frac{\varphi_{2}(\xi) x_{1}(\xi)^{2} n_{1}(\xi)(\xi+1)^{2}-L_{4}^{2,0} r\left(\xi, \mathbf{y}^{q}\right)^{4}}{r\left(\xi, \mathbf{y}^{q}\right)^{4}(\xi+1)^{2}} J(\xi) d \xi+L_{4}^{2,0} \int_{-1}^{1} \frac{1}{(\xi+1)^{2}} d \xi .
\end{gathered}
$$

Integral of the type $J_{4}^{0,2}\left(n_{2}\right)=\int_{-1}^{1} \frac{\varphi_{q}(\xi) x_{2}(\xi)^{2} n_{2}(\xi)}{r\left(\xi, \mathbf{y}^{q}\right)^{4}} J(\xi) d \xi$

Node $q=0$ and $y_{1}^{0}=x_{1}^{0}$

$$
\begin{gathered}
L_{4}^{0,2}=\lim _{\xi \rightarrow-1} \frac{\varphi_{0}(\xi) x_{2}(\xi)^{2} n_{2}(\xi) J(\xi)(\xi-1)^{2}}{r\left(\xi, \mathbf{y}^{q}\right)^{4}}=-\frac{4\left(x_{1}^{0}-x_{1}^{1}\right)\left(l_{2}^{0,2}\right)^{2}}{\left(S_{2}+x_{1}^{2} n_{1}^{0,2}+x_{2}^{2} n_{2}^{0,2}\right)^{2}}, \\
J_{4}^{0,2}\left(n_{1}\right)=\int_{-1}^{1} \frac{\varphi_{0}(\xi) x_{2}(\xi)^{2} n_{2}(\xi)(\xi+1)^{2}-L_{4}^{0,2} r\left(\xi, \mathbf{y}^{q}\right)^{4}}{r\left(\xi, \mathbf{y}^{q}\right)^{4}(\xi+1)^{2}} J(\xi) d \xi+L_{4}^{0,2} \int_{-1}^{1} \frac{1}{(\xi+1)^{2}} d \xi .
\end{gathered}
$$

Node $q=1$ and $y_{1}^{1}=x_{1}^{1}$

$$
\begin{gathered}
L_{4}^{0,2}=\lim _{\xi \rightarrow 0} \frac{\varphi_{1}(\xi) x_{2}(\xi)^{2} n_{2}(\xi) J(\xi) \xi^{2}}{r\left(\xi, \mathbf{y}^{q}\right)^{4}}=\frac{2\left(x_{1}^{0}-x_{1}^{2}\right)\left(x_{2}^{0}-x_{2}^{2}\right)^{2}}{\left(S^{0,2}\right)^{2}}, \\
J_{4}^{0,2}\left(n_{1}\right)=\int_{-1}^{1} \frac{\varphi_{1}(\xi) x_{2}(\xi)^{2} n_{2}(\xi) \xi^{2}-L_{4}^{0,2} r\left(\xi, \mathbf{y}^{q}\right)^{4}}{r\left(\xi, \mathbf{y}^{q}\right)^{4} \xi^{2}} J(\xi) d \xi+L_{4}^{0,2} \int_{-1}^{1} \frac{1}{\xi^{2}} d \xi .
\end{gathered}
$$

Node $q=2$ and $y_{1}^{2}=x_{1}^{2}$

$$
\begin{gathered}
L_{4}^{0,2}=\lim _{\xi \rightarrow-1} \frac{\varphi_{2}(\xi) x_{2}(\xi)^{2} n_{2}(\xi) J(\xi)(\xi-1)^{2}}{r\left(\xi, \mathbf{y}^{q}\right)^{4}}=-\frac{4\left(x_{1}^{2}-x_{1}^{1}\right)\left(l_{2}^{0,2}\right)^{2}}{\left(S_{2}+x_{1}^{2} n_{1}^{0,2}+x_{2}^{2} n_{2}^{0,2}\right)^{2}}, \\
J_{4}^{0,2}\left(n_{1}\right)=\int_{-1}^{1} \frac{\varphi_{2}(\xi) x_{2}(\xi)^{2} n_{2}(\xi)(\xi+1)^{2}-L_{4}^{0,2} r\left(\xi, \mathbf{y}^{q}\right)^{4}}{r\left(\xi, \mathbf{y}^{q}\right)^{4}(\xi+1)^{2}} J(\xi) d \xi+L_{4}^{0,2} \int_{-1}^{1} \frac{1}{(\xi+1)^{2}} d \xi
\end{gathered}
$$

$$
\text { Integral of the type } J_{4}^{1,1}\left(n_{1}\right)=\int_{-1}^{1} \frac{\varphi_{q}(\xi) x_{1}(\xi) x_{2}(\xi) n_{1}(\xi)}{r\left(\xi, \mathbf{y}^{q}\right)^{4}} J(\xi) d \xi
$$

Node $q=0$ and $y_{1}^{0}=x_{1}^{0}$

$$
\begin{gathered}
L_{4}^{1,1}=\lim _{\xi \rightarrow-1} \frac{\varphi_{0}(\xi) x_{1}(\xi) x_{2}(\xi) n_{1}(\xi) J(\xi)(\xi-1)^{2}}{r\left(\xi, \mathbf{y}^{q}\right)^{4}}=-\frac{4\left(x_{2}^{0}-x_{2}^{1}\right) l_{1}^{0,2} l_{2}^{0,2}}{\left(S_{2}+x_{1}^{2} n_{1}^{0,2}+x_{2}^{2} n_{2}^{0,2}\right)^{2}}, \\
J_{4}^{1,1}\left(n_{1}\right)=\int_{-1}^{1} \frac{\varphi_{0}(\xi) x_{1}(\xi) x_{2}(\xi) n_{1}(\xi)(\xi+1)^{2}-L_{4}^{1,1} r\left(\xi, \mathbf{y}^{q}\right)^{4}}{r\left(\xi, \mathbf{y}^{q}\right)^{4}(\xi+1)^{2}} J(\xi) d \xi+L_{4}^{1,1} \int_{-1}^{1} \frac{1}{(\xi+1)^{2}} d \xi .
\end{gathered}
$$


Node $q=1$ and $y_{1}^{1}=x_{1}^{1}$

$$
\begin{aligned}
L_{4}^{1,1} & =\lim _{\xi \rightarrow 0} \frac{\varphi_{1}(\xi) x_{1}(\xi) x_{2}(\xi) n_{1}(\xi) J(\xi) \xi^{2}}{r\left(\xi, \mathbf{y}^{q}\right)^{4}}=-\frac{2\left(x_{1}^{0}-x_{1}^{2}\right)\left(x_{2}^{0}-x_{2}^{2}\right)^{2}}{\left(S^{0,2}\right)^{2}}, \\
J_{4}^{1,1}\left(n_{1}\right) & =\int_{-1}^{1} \frac{\varphi_{1}(\xi) x_{1}(\xi) x_{2}(\xi) n_{1}(\xi) \xi^{2}-L_{4}^{1,1} r\left(\xi, \mathbf{y}^{q}\right)^{4}}{r\left(\xi, \mathbf{y}^{q}\right)^{4} \xi^{2}} J(\xi) d \xi+L_{4}^{1,1} \int_{-1}^{1} \frac{1}{\xi^{2}} d \xi .
\end{aligned}
$$

Node $q=2$ and $y_{1}^{2}=x_{1}^{2}$

$$
\begin{gathered}
L_{4}^{1,1}=\lim _{\xi \rightarrow-1} \frac{\varphi_{2}(\xi) x_{1}(\xi) x_{2}(\xi) n_{1}(\xi) J(\xi)(\xi-1)^{2}}{r\left(\xi, \mathbf{y}^{q}\right)^{4}}=\frac{4\left(x_{2}^{2}-x_{2}^{1}\right) l_{1}^{0,2} l_{2}^{0,2}}{\left(S_{2}+x_{1}^{2} n_{1}^{0,2}+x_{2}^{2} n_{2}^{0,2}\right)^{2}}, \\
J_{4}^{1,1}\left(n_{1}\right)=\int_{-1}^{1} \frac{\varphi_{2}(\xi) x_{1}(\xi) x_{2}(\xi) n_{1}(\xi)(\xi+1)^{2}-L_{4}^{1,1} r\left(\xi, \mathbf{y}^{q}\right)^{4}}{r\left(\xi, \mathbf{y}^{q}\right)^{4}(\xi+1)^{2}} J(\xi) d \xi+L_{4}^{1,1} \int_{-1}^{1} \frac{1}{(\xi+1)^{2}} d \xi .
\end{gathered}
$$

$$
\text { Integral of the type } J_{4}^{1,1}\left(n_{2}\right)=\int_{-1}^{1} \frac{\varphi_{q}(\xi) x_{1}(\xi) x_{2}(\xi) n_{2}(\xi)}{r\left(\xi, \mathbf{y}^{q}\right)^{4}} J(\xi) d \xi
$$

Node $q=0$ and $y_{1}^{0}=x_{1}^{0}$

$$
\begin{gathered}
L_{4}^{1,1}=\lim _{\xi \rightarrow-1} \frac{\varphi_{0}(\xi) x_{1}(\xi) x_{2}(\xi) n_{2}(\xi) J(\xi)(\xi-1)^{2}}{r\left(\xi, \mathbf{y}^{q}\right)^{4}}=\frac{4\left(x_{1}^{0}-x_{1}^{1}\right) l_{1}^{0,2} l_{2}^{0,2}}{\left(S_{2}+x_{1}^{2} n_{1}^{0,2}+x_{2}^{2} n_{2}^{0,2}\right)^{2}}, \\
J_{4}^{1,1}\left(n_{2}\right)=\int_{-1}^{1} \frac{\varphi_{0}(\xi) x_{1}(\xi) x_{2}(\xi) n_{2}(\xi)(\xi+1)^{2}-L_{4}^{1,1} r\left(\xi, \mathbf{y}^{q}\right)^{4}}{r\left(\xi, \mathbf{y}^{q}\right)^{4}(\xi+1)^{2}} J(\xi) d \xi+L_{4}^{1,1} \int_{-1}^{1} \frac{1}{(\xi+1)^{2}} d \xi .
\end{gathered}
$$

Node $q=1$ and $y_{1}^{1}=x_{1}^{1}$

$$
\begin{aligned}
L_{4}^{1,1} & =\lim _{\xi \rightarrow 0} \frac{\varphi_{1}(\xi) x_{1}(\xi) x_{2}(\xi) n_{2}(\xi) J(\xi) \xi^{2}}{r\left(\xi, \mathbf{y}^{q}\right)^{4}}=\frac{2\left(x_{1}^{0}-x_{1}^{2}\right)^{2}\left(x_{2}^{0}-x_{2}^{2}\right)}{\left(S^{0,2}\right)^{2}}, \\
J_{4}^{1,1}\left(n_{2}\right) & =\int_{-1}^{1} \frac{\varphi_{1}(\xi) x_{1}(\xi) x_{2}(\xi) n_{2}(\xi) \xi^{2}-L_{4}^{1,1} r\left(\xi, \mathbf{y}^{q}\right)^{4}}{r\left(\xi, \mathbf{y}^{q}\right)^{4} \xi^{2}} J(\xi) d \xi+L_{4}^{1,1} \int_{-1}^{1} \frac{1}{\xi^{2}} d \xi .
\end{aligned}
$$

Node $q=2$ and $y_{1}^{2}=x_{1}^{2}$

$$
\begin{aligned}
L_{4}^{1,1} & =\lim _{\xi \rightarrow-1} \frac{\varphi_{2}(\xi) x_{1}(\xi) x_{2}(\xi) n_{2}(\xi) J(\xi)(\xi-1)^{2}}{r\left(\xi, \mathbf{y}^{q}\right)^{4}}=-\frac{4\left(x_{1}^{2}-x_{1}^{1}\right) l_{1}^{0,2} l_{2}^{0,2}}{\left(S_{2}+x_{1}^{2} n_{1}^{0,2}+x_{2}^{2} n_{2}^{0,2}\right)^{2}}, \\
J_{4}^{1,1}\left(n_{2}\right) & =\int_{-1}^{1} \frac{\varphi_{2}(\xi) x_{1}(\xi) x_{2}(\xi) n_{2}(\xi)(\xi+1)^{2}-L_{4}^{1,1} r\left(\xi, \mathbf{y}^{q}\right)^{4}}{r\left(\xi, \mathbf{y}^{q}\right)^{4}(\xi+1)^{2}} J(\xi) d \xi+L_{4}^{1,1} \int_{-1}^{1} \frac{1}{(\xi+1)^{2}} d \xi .
\end{aligned}
$$




$$
\text { Integral of the type } J_{6}^{4,0}\left(n_{1}\right)=\int_{-1}^{1} \frac{\varphi_{q}(\xi) x_{1}(\xi)^{4} n_{1}(\xi)}{r\left(\xi, \mathbf{y}^{q}\right)^{6}} J(\xi) d \xi
$$

Node $q=0$ and $y_{1}^{0}=x_{1}^{0}$

$$
\begin{gathered}
L_{6}^{4,0}=\lim _{\xi \rightarrow-1} \frac{\varphi_{0}(\xi) x_{1}(\xi)^{6} n_{1}(\xi) J(\xi)(\xi-1)^{2}}{r\left(\xi, \mathbf{y}^{q}\right)^{6}}=-\frac{4\left(x_{2}^{0}-x_{2}^{1}\right)\left(l_{1}^{0,2}\right)^{4}}{\left(S_{2}+x_{1}^{2} n_{1}^{0,2}+x_{2}^{2} n_{2}^{0,2}\right)^{3}}, \\
J_{6}^{4,0}\left(n_{1}\right)=\int_{-1}^{1} \frac{\varphi_{0}(\xi) x_{1}(\xi)^{4} n_{1}(\xi)(\xi+1)^{2}-L_{4}^{2,0} r\left(\xi, \mathbf{y}^{q}\right)^{6}}{r\left(\xi, \mathbf{y}^{q}\right)^{6}(\xi+1)^{2}} J(\xi) d \xi+L_{6}^{4,0} \int_{-1}^{1} \frac{1}{(\xi+1)^{2}} d \xi .
\end{gathered}
$$

Node $q=1$ and $y_{1}^{1}=x_{1}^{1}$

$$
\begin{gathered}
L_{4}^{2,0}=\lim _{\xi \rightarrow 0} \frac{\varphi_{1}(\xi) x_{1}(\xi)^{2} n_{1}(\xi) J(\xi) \xi^{2}}{r\left(\xi, \mathbf{y}^{q}\right)^{4}}=-\frac{2\left(x_{1}^{0}-x_{1}^{2}\right)^{4}\left(x_{2}^{0}-x_{2}^{2}\right)}{\left(S^{0,2}\right)^{3}}, \\
J_{6}^{4,0}\left(n_{1}\right)=\int_{-1}^{1} \frac{\varphi_{1}(\xi) x_{1}(\xi)^{4} n_{1}(\xi) \xi^{2}-L_{6}^{4,0} r\left(\xi, \mathbf{y}^{q}\right)^{6}}{r\left(\xi, \mathbf{y}^{q}\right)^{6} \xi^{2}} J(\xi) d \xi+L_{6}^{4,0} \int_{-1}^{1} \frac{1}{\xi^{2}} d \xi .
\end{gathered}
$$

Node $q=2$ and $y_{1}^{2}=x_{1}^{2}$

$$
\begin{gathered}
L_{6}^{4,0}=\lim _{\xi \rightarrow-1} \frac{\varphi_{2}(\xi) x_{1}(\xi)^{4} n_{1}(\xi) J(\xi)(\xi-1)^{2}}{r\left(\xi, \mathbf{y}^{q}\right)^{6}}=\frac{4\left(x_{2}^{2}-x_{2}^{1}\right)\left(l_{1}^{0,2}\right)^{4}}{\left(S_{2}+x_{1}^{2} n_{1}^{0,2}+x_{2}^{2} n_{2}^{0,2}\right)^{3}}, \\
J_{6}^{4,0}\left(n_{1}\right)=\int_{-1}^{1} \frac{\varphi_{2}(\xi) x_{1}(\xi)^{4} n_{1}(\xi)(\xi+1)^{2}-L_{6}^{4,0} r\left(\xi, \mathbf{y}^{q}\right)^{6}}{r\left(\xi, \mathbf{y}^{q}\right)^{6}(\xi+1)^{2}} J(\xi) d \xi+L_{6}^{4,0} \int_{-1}^{1} \frac{1}{(\xi+1)^{2}} d \xi .
\end{gathered}
$$

Integral of the type $J_{6}^{0,4}\left(n_{2}\right)=\int_{-1}^{1} \frac{\varphi_{q}(\xi) x_{2}(\xi)^{4} n_{2}(\xi)}{r\left(\xi, \mathbf{y}^{q}\right)^{6}} J(\xi) d \xi$

Node $q=0$ and $y_{1}^{0}=x_{1}^{0}$

$$
\begin{gathered}
L_{6}^{0,4}=\lim _{\xi \rightarrow-1} \frac{\varphi_{0}(\xi) x_{2}(\xi)^{6} n_{2}(\xi) J(\xi)(\xi-1)^{2}}{r\left(\xi, \mathbf{y}^{q}\right)^{6}}=\frac{4\left(x_{1}^{0}-x_{1}^{1}\right)\left(l_{1}^{0,2}\right)^{4}}{\left(S_{2}+x_{1}^{2} n_{1}^{0,2}+x_{2}^{2} n_{2}^{0,2}\right)^{3}}, \\
J_{6}^{0,4}\left(n_{2}\right)=\int_{-1}^{1} \frac{\varphi_{0}(\xi) x_{2}(\xi)^{4} n_{2}(\xi)(\xi+1)^{2}-L_{6}^{0,4} r\left(\xi, \mathbf{y}^{q}\right)^{6}}{r\left(\xi, \mathbf{y}^{q}\right)^{6}(\xi+1)^{2}} J(\xi) d \xi+L_{6}^{0,4} \int_{-1}^{1} \frac{1}{(\xi+1)^{2}} d \xi .
\end{gathered}
$$

Node $q=1$ and $y_{1}^{1}=x_{1}^{1}$

$$
\begin{gathered}
L_{6}^{0,4}=\lim _{\xi \rightarrow 0} \frac{\varphi_{1}(\xi) x_{1}(\xi)^{2} n_{1}(\xi) J(\xi) \xi^{2}}{r\left(\xi, \mathbf{y}^{q}\right)^{4}}=\frac{2\left(x_{1}^{0}-x_{1}^{2}\right)\left(x_{2}^{0}-x_{2}^{2}\right)^{4}}{\left(S^{0,2}\right)^{3}}, \\
J_{6}^{0,4}\left(n_{2}\right)=\int_{-1}^{1} \frac{\varphi_{1}(\xi) x_{2}(\xi)^{4} n_{2}(\xi) \xi^{2}-L_{6}^{0,4} r\left(\xi, \mathbf{y}^{q}\right)^{6}}{r\left(\xi, \mathbf{y}^{q}\right)^{6} \xi^{2}} J(\xi) d \xi+L_{6}^{0,4} \int_{-1}^{1} \frac{1}{\xi^{2}} d \xi .
\end{gathered}
$$

Node $q=2$ and $y_{1}^{2}=x_{1}^{2}$

$$
L_{6}^{0,4}=\lim _{\xi \rightarrow-1} \frac{\varphi_{2}(\xi) x_{2}(\xi)^{4} n_{2}(\xi) J(\xi)(\xi-1)^{2}}{r\left(\xi, \mathbf{y}^{q}\right)^{6}}=\frac{4\left(x_{1}^{2}-x_{1}^{1}\right)\left(l_{2}^{0,2}\right)^{4}}{\left(S_{2}+x_{1}^{2} n_{1}^{0,2}+x_{2}^{2} n_{2}^{0,2}\right)^{3}},
$$




$$
J_{6}^{0,4}\left(n_{2}\right)=\int_{-1}^{1} \frac{\varphi_{2}(\xi) x_{2}(\xi)^{4} n_{2}(\xi)(\xi+1)^{2}-L_{6}^{0,4} r\left(\xi, \mathbf{y}^{q}\right)^{6}}{r\left(\xi, \mathbf{y}^{q}\right)^{6}(\xi+1)^{2}} J(\xi) d \xi+L_{6}^{0,4} \int_{-1}^{1} \frac{1}{(\xi+1)^{2}} d \xi
$$

$$
\text { Integral of the type } J_{6}^{3,1}\left(n_{1}\right)=\int_{-1}^{1} \frac{\varphi_{q}(\xi) x_{1}(\xi)^{3} x_{2}(\xi) n_{1}(\xi)}{r\left(\xi, \mathbf{y}^{q}\right)^{6}} J(\xi) d \xi
$$

Node $q=0$ and $y_{1}^{0}=x_{1}^{0}$

$$
\begin{aligned}
L_{6}^{3,1} & =\lim _{\xi \rightarrow-1} \frac{\varphi_{0}(\xi) x_{1}(\xi)^{3} x_{2}(\xi) n_{1}(\xi) J(\xi)(\xi-1)^{2}}{r\left(\xi, \mathbf{y}^{q}\right)^{6}}=-\frac{4\left(x_{2}^{0}-x_{2}^{1}\right)\left(l_{1}^{0,2}\right)^{3} l_{2}^{0,2}}{\left(S_{2}+x_{1}^{2} n_{1}^{0,2}+x_{2}^{2} n_{2}^{0,2}\right)^{3}}, \\
J_{6}^{3,1}\left(n_{1}\right) & =\int_{-1}^{1} \frac{\varphi_{0}(\xi) x_{1}(\xi)^{3} x_{2}(\xi) n_{1}(\xi)(\xi+1)^{2}-L_{6}^{3,1} r\left(\xi, \mathbf{y}^{q}\right)^{6}}{r\left(\xi, \mathbf{y}^{q}\right)^{6}(\xi+1)^{2}} J(\xi) d \xi+L_{6}^{3,1} \int_{-1}^{1} \frac{1}{(\xi+1)^{2}} d \xi .
\end{aligned}
$$

Node $q=1$ and $y_{1}^{1}=x_{1}^{1}$

$$
\begin{gathered}
L_{6}^{3,1}=\lim _{\xi \rightarrow 0} \frac{\varphi_{1}(\xi) x_{1}(\xi)^{3} x_{2}(\xi) n_{1}(\xi) J(\xi) \xi^{2}}{r\left(\xi, \mathbf{y}^{q}\right)^{6}}=-\frac{2\left(x_{1}^{0}-x_{1}^{2}\right)^{3}\left(x_{2}^{0}-x_{2}^{2}\right)^{2}}{\left(S^{0,2}\right)^{3}}, \\
J_{6}^{3,1}\left(n_{1}\right)=\int_{-1}^{1} \frac{\varphi_{1}(\xi) x_{1}(\xi)^{3} x_{2}(\xi) n_{1}(\xi) \xi^{2}-L_{6}^{3,1} r\left(\xi, \mathbf{y}^{q}\right)^{6}}{r\left(\xi, \mathbf{y}^{q}\right)^{6} \xi^{2}} J(\xi) d \xi+L_{6}^{3,1} \int_{-1}^{1} \frac{1}{\xi^{2}} d \xi .
\end{gathered}
$$

Node $q=2$ and $y_{1}^{2}=x_{1}^{2}$

$$
\begin{gathered}
L_{6}^{3,1}=\lim _{\xi \rightarrow-1} \frac{\varphi_{2}(\xi) x_{1}(\xi)^{3} x_{2}(\xi) n_{1}(\xi) J(\xi)(\xi-1)^{2}}{r\left(\xi, \mathbf{y}^{q}\right)^{6}}=\frac{4\left(x_{2}^{2}-x_{2}^{1}\right)\left(l_{1}^{0,2}\right)^{3} l_{2}^{0,2}}{\left(S_{2}+x_{1}^{2} n_{1}^{0,2}+x_{2}^{2} n_{2}^{0,2}\right)^{3}} \\
J_{6}^{3,1}\left(n_{1}\right)=\int_{-1}^{1} \frac{\varphi_{2}(\xi) x_{1}(\xi)^{3} x_{2}(\xi) n_{1}(\xi)(\xi+1)^{2}-L_{6}^{3,1} r\left(\xi, \mathbf{y}^{q}\right)^{6}}{r\left(\xi, \mathbf{y}^{q}\right)^{6}(\xi+1)^{2}} J(\xi) d \xi+L_{6}^{3,1} \int_{-1}^{1} \frac{1}{(\xi+1)^{2}} d \xi .
\end{gathered}
$$

Integral of the type $J_{6}^{3,1}\left(n_{2}\right)=\int_{-1}^{1} \frac{\varphi_{q}(\xi) x_{1}(\xi)^{3} x_{2}(\xi) n_{2}(\xi)}{r\left(\xi, \mathbf{y}^{q}\right)^{6}} J(\xi) d \xi$

Node $q=0$ and $y_{1}^{0}=x_{1}^{0}$

$$
\begin{gathered}
L_{6}^{3,1}=\lim _{\xi \rightarrow-1} \frac{\varphi_{0}(\xi) x_{1}(\xi)^{3} x_{2}(\xi) n_{2}(\xi) J(\xi)(\xi-1)^{2}}{r\left(\xi, \mathbf{y}^{q}\right)^{6}}=\frac{4\left(x_{1}^{0}-x_{1}^{1}\right)\left(l_{1}^{0,2}\right)^{3} l_{2}^{0,2}}{\left(S_{2}+x_{1}^{2} n_{1}^{0,2}+x_{2}^{2} n_{2}^{0,2}\right)^{3}}, \\
J_{6}^{3,1}\left(n_{2}\right)=\int_{-1}^{1} \frac{\varphi_{0}(\xi) x_{1}(\xi)^{3} x_{2}(\xi) n_{2}(\xi)(\xi+1)^{2}-L_{6}^{3,1} r\left(\xi, \mathbf{y}^{q}\right)^{6}}{r\left(\xi, \mathbf{y}^{q}\right)^{6}(\xi+1)^{2}} J(\xi) d \xi+L_{6}^{3,1} \int_{-1}^{1} \frac{1}{(\xi+1)^{2}} d \xi .
\end{gathered}
$$

Node $q=1$ and $y_{1}^{1}=x_{1}^{1}$

$$
\begin{aligned}
L_{6}^{3,1} & =\lim _{\xi \rightarrow 0} \frac{\varphi_{1}(\xi) x_{1}(\xi)^{3} x_{2}(\xi) n_{2}(\xi) J(\xi) \xi^{2}}{r\left(\xi, \mathbf{y}^{q}\right)^{6}}=\frac{2\left(x_{1}^{0}-x_{1}^{2}\right)^{4}\left(x_{2}^{0}-x_{2}^{2}\right)}{\left(S^{0,2}\right)^{3}}, \\
J_{6}^{3,1}\left(n_{1}\right) & =\int_{-1}^{1} \frac{\varphi_{1}(\xi) x_{1}(\xi)^{3} x_{2}(\xi) n_{2}(\xi) \xi^{2}-L_{6}^{3,1} r\left(\xi, \mathbf{y}^{q}\right)^{6}}{r\left(\xi, \mathbf{y}^{q}\right)^{6} \xi^{2}} J(\xi) d \xi+L_{6}^{3,1} \int_{-1}^{1} \frac{1}{\xi^{2}} d \xi .
\end{aligned}
$$


Node $q=2$ and $y_{1}^{2}=x_{1}^{2}$

$$
\begin{gathered}
L_{6}^{3,1}=\lim _{\xi \rightarrow-1} \frac{\varphi_{2}(\xi) x_{1}(\xi)^{3} x_{2}(\xi) n_{2}(\xi) J(\xi)(\xi-1)^{2}}{r\left(\xi, \mathbf{y}^{q}\right)^{6}}=-\frac{4\left(x_{1}^{2}-x_{1}^{1}\right)\left(l_{1}^{0,2}\right)^{3} l_{2}^{0,2}}{\left(S_{2}+x_{1}^{2} n_{1}^{0,2}+x_{2}^{2} n_{2}^{0,2}\right)^{3}}, \\
J_{6}^{3,1}\left(n_{2}\right)=\int_{-1}^{1} \frac{\varphi_{2}(\xi) x_{1}(\xi)^{3} x_{2}(\xi) n_{2}(\xi)(\xi+1)^{2}-L_{6}^{3,1} r\left(\xi, \mathbf{y}^{q}\right)^{6}}{r\left(\xi, \mathbf{y}^{q}\right)^{6}(\xi+1)^{2}} J(\xi) d \xi+L_{6}^{3,1} \int_{-1}^{1} \frac{1}{(\xi+1)^{2}} d \xi .
\end{gathered}
$$

Integral of the type $J_{6}^{2,2}\left(n_{1}\right)=\int_{-1}^{1} \frac{\varphi_{q}(\xi) x_{1}(\xi)^{2} x_{2}(\xi)^{2} n_{1}(\xi)}{r\left(\xi, \mathbf{y}^{q}\right)^{6}} J(\xi) d \xi$

Node $q=0$ and $y_{1}^{0}=x_{1}^{0}$

$$
\begin{gathered}
L_{6}^{2,2}=\lim _{\xi \rightarrow-1} \frac{\varphi_{0}(\xi) x_{1}(\xi)^{2} x_{2}(\xi)^{2} n_{1}(\xi) J(\xi)(\xi-1)^{2}}{r\left(\xi, \mathbf{y}^{q}\right)^{6}}=-\frac{4\left(x_{2}^{0}-x_{2}^{1}\right)\left(l_{1}^{0,2}\right)^{2}\left(l_{2}^{0,2}\right)^{2}}{\left(S_{2}+x_{1}^{2} n_{1}^{0,2}+x_{2}^{2} n_{2}^{0,2}\right)^{3}}, \\
J_{6}^{2,2}\left(n_{1}\right)=\int_{-1}^{1} \frac{\varphi_{0}(\xi) x_{1}(\xi)^{2} x_{2}(\xi)^{2} n_{1}(\xi)(\xi+1)^{2}-L_{6}^{2,2} r\left(\xi, \mathbf{y}^{q}\right)^{6}}{r\left(\xi, \mathbf{y}^{q}\right)^{6}(\xi+1)^{2}} J(\xi) d \xi+L_{6}^{2,2} \int_{-1}^{1} \frac{1}{(\xi+1)^{2}} d \xi .
\end{gathered}
$$

Node $q=1$ and $y_{1}^{1}=x_{1}^{1}$

$$
\begin{aligned}
L_{6}^{2,2} & =\lim _{\xi \rightarrow 0} \frac{\varphi_{1}(\xi) x_{1}(\xi)^{2} x_{2}(\xi)^{2} n_{1}(\xi) J(\xi) \xi^{2}}{r\left(\xi, \mathbf{y}^{q}\right)^{6}}=-\frac{2\left(x_{1}^{0}-x_{1}^{2}\right)^{2}\left(x_{2}^{0}-x_{2}^{2}\right)^{3}}{\left(S^{0,2}\right)^{3}}, \\
J_{6}^{2,2}\left(n_{1}\right) & =\int_{-1}^{1} \frac{\varphi_{1}(\xi) x_{1}(\xi)^{2} x_{2}(\xi)^{2} n_{1}(\xi) \xi^{2}-L_{6}^{2,2} r\left(\xi, \mathbf{y}^{q}\right)^{6}}{r\left(\xi, \mathbf{y}^{q}\right)^{6} \xi^{2}} J(\xi) d \xi+L_{6}^{2,2} \int_{-1}^{1} \frac{1}{\xi^{2}} d \xi .
\end{aligned}
$$

Node $q=2$ and $y_{1}^{2}=x_{1}^{2}$

$$
\begin{gathered}
L_{6}^{2,2}=\lim _{\xi \rightarrow-1} \frac{\varphi_{2}(\xi) x_{1}(\xi)^{2} x_{2}(\xi)^{2} n_{1}(\xi) J(\xi)(\xi-1)^{2}}{r\left(\xi, \mathbf{y}^{q}\right)^{6}}=\frac{4\left(x_{2}^{2}-x_{2}^{1}\right)\left(l_{1}^{0,2}\right)^{2}\left(l_{2}^{0,2}\right)^{2}}{\left(S_{2}+x_{1}^{2} n_{1}^{0,2}+x_{2}^{2} n_{2}^{0,2}\right)^{3}} \\
J_{6}^{2,2}\left(n_{1}\right)=\int_{-1}^{1} \frac{\varphi_{2}(\xi) x_{1}(\xi)^{2} x_{2}(\xi)^{2} n_{1}(\xi)(\xi+1)^{2}-L_{6}^{2,2} r\left(\xi, \mathbf{y}^{q}\right)^{6}}{r\left(\xi, \mathbf{y}^{q}\right)^{6}(\xi+1)^{2}} J(\xi) d \xi+L_{6}^{2,2} \int_{-1}^{1} \frac{1}{(\xi+1)^{2}} d \xi .
\end{gathered}
$$

$$
\text { Integral of the type } J_{6}^{2,2}\left(n_{2}\right)=\int_{-1}^{1} \frac{\varphi_{q}(\xi) x_{1}(\xi)^{2} x_{2}(\xi)^{2} n_{2}(\xi)}{r\left(\xi, \mathbf{y}^{q}\right)^{6}} J(\xi) d \xi
$$

Node $q=0$ and $y_{1}^{0}=x_{1}^{0}$

$$
\begin{aligned}
& L_{6}^{2,2}=\lim _{\xi \rightarrow-1} \frac{\varphi_{0}(\xi) x_{1}(\xi)^{2} x_{2}(\xi)^{2} n_{2}(\xi) J(\xi)(\xi-1)^{2}}{r\left(\xi, \mathbf{y}^{q}\right)^{6}}=\frac{4\left(x_{1}^{0}-x_{1}^{1}\right)\left(l_{1}^{0,2}\right)^{2}\left(l_{2}^{0,2}\right)^{2}}{\left(S_{2}+x_{1}^{2} n_{1}^{0,2}+x_{2}^{2} n_{2}^{0,2}\right)^{3}}, \\
& J_{6}^{2,2}\left(n_{2}\right)=\int_{-1}^{1} \frac{\varphi_{0}(\xi) x_{1}(\xi)^{2} x_{2}(\xi)^{2} n_{2}(\xi)(\xi+1)^{2}-L_{6}^{2,2} r\left(\xi, \mathbf{y}^{q}\right)^{6}}{r\left(\xi, \mathbf{y}^{q}\right)^{6}(\xi+1)^{2}} J(\xi) d \xi+L_{6}^{2,2} \int_{-1}^{1} \frac{1}{(\xi+1)^{2}} d \xi .
\end{aligned}
$$

Node $q=1$ and $y_{1}^{1}=x_{1}^{1}$ 


$$
\begin{aligned}
L_{6}^{2,2} & =\lim _{\xi \rightarrow 0} \frac{\varphi_{1}(\xi) x_{1}(\xi)^{2} x_{2}(\xi)^{2} n_{2}(\xi) J(\xi) \xi^{2}}{r\left(\xi, \mathbf{y}^{q}\right)^{6}}=\frac{2\left(x_{1}^{0}-x_{1}^{2}\right)^{3}\left(x_{2}^{0}-x_{2}^{2}\right)^{2}}{\left(S^{0,2}\right)^{3}}, \\
J_{6}^{2,2}\left(n_{2}\right) & =\int_{-1}^{1} \frac{\varphi_{1}(\xi) x_{1}(\xi)^{2} x_{2}(\xi)^{2} n_{2}(\xi) \xi^{2}-L_{6}^{2,2} r\left(\xi, \mathbf{y}^{q}\right)^{6}}{r\left(\xi, \mathbf{y}^{q}\right)^{6} \xi^{2}} J(\xi) d \xi+L_{6}^{2,2} \int_{-1}^{1} \frac{1}{\xi^{2}} d \xi .
\end{aligned}
$$

Node $q=2$ and $y_{1}^{2}=x_{1}^{2}$

$$
\begin{gathered}
L_{6}^{2,2}=\lim _{\xi \rightarrow-1} \frac{\varphi_{2}(\xi) x_{1}(\xi)^{2} x_{2}(\xi)^{2} n_{2}(\xi) J(\xi)(\xi-1)^{2}}{r\left(\xi, \mathbf{y}^{q}\right)^{6}}=-\frac{4\left(x_{1}^{2}-x_{1}^{1}\right)\left(l_{1}^{0,2}\right)^{2}\left(l_{2}^{0,2}\right)^{2}}{\left(S_{2}+x_{1}^{2} n_{1}^{0,2}+x_{2}^{2} n_{2}^{0,2}\right)^{3}}, \\
J_{6}^{2,2}\left(n_{2}\right)=\int_{-1}^{1} \frac{\varphi_{2}(\xi) x_{1}(\xi)^{2} x_{2}(\xi)^{2} n_{2}(\xi)(\xi+1)^{2}-L_{6}^{2,2} r\left(\xi, \mathbf{y}^{q}\right)^{6}}{r\left(\xi, \mathbf{y}^{q}\right)^{6}(\xi+1)^{2}} J(\xi) d \xi+L_{6}^{2,2} \int_{-1}^{1} \frac{1}{(\xi+1)^{2}} d \xi .
\end{gathered}
$$

$$
\text { Integral of the type } J_{6}^{1,3}\left(n_{1}\right)=\int_{-1}^{1} \frac{\varphi_{q}(\xi) x_{1}(\xi) x_{2}(\xi)^{3} n_{1}(\xi)}{r\left(\xi, \mathbf{y}^{q}\right)^{6}} J(\xi) d \xi
$$

Node $q=0$ and $y_{1}^{0}=x_{1}^{0}$

$$
\begin{gathered}
L_{6}^{1,3}=\lim _{\xi \rightarrow-1} \frac{\varphi_{0}(\xi) x_{1}(\xi) x_{2}(\xi)^{3} n_{1}(\xi) J(\xi)(\xi-1)^{2}}{r\left(\xi, \mathbf{y}^{q}\right)^{6}}=-\frac{4\left(x_{2}^{0}-x_{2}^{1}\right) l_{1}^{0,2}\left(l_{2}^{0,2}\right)^{3}}{\left(S_{2}+x_{1}^{2} n_{1}^{0,2}+x_{2}^{2} n_{2}^{0,2}\right)^{3}}, \\
J_{6}^{1,3}\left(n_{1}\right)=\int_{-1}^{1} \frac{\varphi_{0}(\xi) x_{1}(\xi) x_{2}(\xi)^{3} n_{1}(\xi)(\xi+1)^{2}-L_{6}^{1,3} r\left(\xi, \mathbf{y}^{q}\right)^{6}}{r\left(\xi, \mathbf{y}^{q}\right)^{6}(\xi+1)^{2}} J(\xi) d \xi+L_{6}^{1,3} \int_{-1}^{1} \frac{1}{(\xi+1)^{2}} d \xi .
\end{gathered}
$$

Node $q=1$ and $y_{1}^{1}=x_{1}^{1}$

$$
\begin{aligned}
L_{6}^{1,3} & =\lim _{\xi \rightarrow 0} \frac{\varphi_{1}(\xi) x_{1}(\xi)^{3} x_{2}(\xi) n_{1}(\xi) J(\xi) \xi^{2}}{r\left(\xi, \mathbf{y}^{q}\right)^{6}}=-\frac{2\left(x_{1}^{0}-x_{1}^{2}\right)\left(x_{2}^{0}-x_{2}^{2}\right)^{4}}{\left(S^{0,2}\right)^{3}}, \\
J_{6}^{1,3}\left(n_{1}\right) & =\int_{-1}^{1} \frac{\varphi_{1}(\xi) x_{1}(\xi) x_{2}(\xi)^{3} n_{1}(\xi) \xi^{2}-L_{6}^{1,3} r\left(\xi, \mathbf{y}^{q}\right)^{6}}{r\left(\xi, \mathbf{y}^{q}\right)^{6} \xi^{2}} J(\xi) d \xi+L_{6}^{1,3} \int_{-1}^{1} \frac{1}{\xi^{2}} d \xi .
\end{aligned}
$$

Node $q=2$ and $y_{1}^{2}=x_{1}^{2}$

$$
\begin{gathered}
L_{6}^{1,3}=\lim _{\xi \rightarrow-1} \frac{\varphi_{2}(\xi) x_{1}(\xi) x_{2}(\xi)^{3} n_{1}(\xi) J(\xi)(\xi-1)^{2}}{r\left(\xi, \mathbf{y}^{q}\right)^{6}}=\frac{4\left(x_{2}^{2}-x_{2}^{1}\right) l_{1}^{0,2}\left(l_{2}^{0,2}\right)^{3}}{\left(S_{2}+x_{1}^{2} n_{1}^{0,2}+x_{2}^{2} n_{2}^{0,2}\right)^{3}}, \\
J_{6}^{1,3}\left(n_{1}\right)=\int_{-1}^{1} \frac{\varphi_{2}(\xi) x_{1}(\xi) x_{2}(\xi)^{3} n_{1}(\xi)(\xi+1)^{2}-L_{6}^{1,3} r\left(\xi, \mathbf{y}^{q}\right)^{6}}{r\left(\xi, \mathbf{y}^{q}\right)^{6}(\xi+1)^{2}} J(\xi) d \xi+L_{6}^{1,3} \int_{-1}^{1} \frac{1}{(\xi+1)^{2}} d \xi .
\end{gathered}
$$

Integral of the type $J_{6}^{1,3}\left(n_{2}\right)=\int_{-1}^{1} \frac{\varphi_{q}(\xi) x_{1}(\xi) x_{2}(\xi)^{3} n_{2}(\xi)}{r\left(\xi, \mathbf{y}^{q}\right)^{6}} J(\xi) d \xi$

Node $q=0$ and $y_{1}^{0}=x_{1}^{0}$

$$
L_{6}^{1,3}=\lim _{\xi \rightarrow-1} \frac{\varphi_{0}(\xi) x_{1}(\xi) x_{2}(\xi)^{3} n_{2}(\xi) J(\xi)(\xi-1)^{2}}{r\left(\xi, \mathbf{y}^{q}\right)^{6}}=\frac{4\left(x_{1}^{0}-x_{1}^{1}\right) l_{1}^{0,2}\left(l_{2}^{0,2}\right)^{3}}{\left(S_{2}+x_{1}^{2} n_{1}^{0,2}+x_{2}^{2} n_{2}^{0,2}\right)^{3}},
$$




$$
J_{6}^{1,3}\left(n_{2}\right)=\int_{-1}^{1} \frac{\varphi_{0}(\xi) x_{1}(\xi) x_{2}(\xi)^{3} n_{2}(\xi)(\xi+1)^{2}-L_{6}^{1,3} r\left(\xi, \mathbf{y}^{q}\right)^{6}}{r\left(\xi, \mathbf{y}^{q}\right)^{6}(\xi+1)^{2}} J(\xi) d \xi+L_{6}^{1,3} \int_{-1}^{1} \frac{1}{(\xi+1)^{2}} d \xi .
$$

Node $q=1$ and $y_{1}^{1}=x_{1}^{1}$

$$
\begin{aligned}
L_{6}^{1,3} & =\lim _{\xi \rightarrow 0} \frac{\varphi_{1}(\xi) x_{1}(\xi) x_{2}(\xi)^{3} n_{2}(\xi) J(\xi) \xi^{2}}{r\left(\xi, \mathbf{y}^{q}\right)^{6}}=\frac{2\left(x_{1}^{0}-x_{1}^{2}\right)^{2}\left(x_{2}^{0}-x_{2}^{2}\right)^{3}}{\left(S^{0,2}\right)^{3}} \\
J_{6}^{1,3}\left(n_{2}\right) & =\int_{-1}^{1} \frac{\varphi_{1}(\xi) x_{1}(\xi)^{3} x_{2}(\xi) n_{2}(\xi) \xi^{2}-L_{6}^{1,3} r\left(\xi, \mathbf{y}^{q}\right)^{6}}{r\left(\xi, \mathbf{y}^{q}\right)^{6} \xi^{2}} J(\xi) d \xi+L_{6}^{1,3} \int_{-1}^{1} \frac{1}{\xi^{2}} d \xi .
\end{aligned}
$$

Node $q=2$ and $y_{1}^{2}=x_{1}^{2}$

$$
\begin{gathered}
L_{6}^{1,3}=\lim _{\xi \rightarrow-1} \frac{\varphi_{2}(\xi) x_{1}(\xi) x_{2}(\xi)^{3} n_{2}(\xi) J(\xi)(\xi-1)^{2}}{r\left(\xi, \mathbf{y}^{q}\right)^{6}}=-\frac{4\left(x_{1}^{2}-x_{1}^{1}\right) l_{1}^{0,2}\left(l_{2}^{0,2}\right)^{3}}{\left(S_{2}+x_{1}^{2} n_{1}^{0,2}+x_{2}^{2} n_{2}^{0,2}\right)^{3}} \\
J_{6}^{1,3}\left(n_{2}\right)=\int_{-1}^{1} \frac{\varphi_{2}(\xi) x_{1}(\xi) x_{2}(\xi)^{3} n_{2}(\xi)(\xi+1)^{2}-L_{6}^{1,3} r\left(\xi, \mathbf{y}^{q}\right)^{6}}{r\left(\xi, \mathbf{y}^{q}\right)^{6}(\xi+1)^{2}} J(\xi) d \xi+L_{6}^{1,3} \int_{-1}^{1} \frac{1}{(\xi+1)^{2}} d \xi .
\end{gathered}
$$

Here $S_{2}=\left(3 x_{1}^{1}-4 x_{1}^{2}\right)^{2}+\left(3 x_{2}^{1}-4 x_{2}^{2}\right)^{2}, n_{i}^{\alpha, \beta}=6 x_{i}^{\alpha}-8 x_{i}^{1}+x_{i}^{\beta}$. 